\title{
Equation of state driven radiative models for simulation of lightning strikes
}

\author{
M. Apsley, ${ }^{1, a)}$ S. T. Millmore, ${ }^{1}$ and N. Nikiforakis ${ }^{1}$ \\ Cavendish Laboratory, Department of Physics, University of Cambridge, Cambridge, United Kingdom
}

(Dated: 12 July 2021)

This work is concerned with the numerical simulation of plasma arc interaction with aerospace substrates under conditions akin to lightning strike and in particular with the accurate calculation of radiative heat losses. These are important because they have a direct effect on the calculation of thermal and pressure loads on the substrates, which can lead to material damage under certain conditions. Direct numerical solution of the radiation transport equation (RTE) in mesoscale simulations is not viable due to its computational cost, so for practical applications reduced models are usually employed. To this end, four approximations for solving the RTE are considered in this work, ranging from a simple local thermodynamical behaviour consideration, to a more complex spectral absorption dependent on the arc geometry. Their performance is initially tested on a one-dimensional cylindrical arc, before implementing them in a multi-dimensional magnetohydrodynamics code. Results indicate that inclusion of spectral absorption is necessary in order to obtain consistent results. However, the approaches accounting for the arc geometry require repeated solution of the computationally intensive Helmholtz equations, making them prohibitive for multi-dimensional simulations. As an alternative, a method using the net emission coefficient is employed, which provides a balance between computational efficiency and accuracy, as shown by comparisons against experimental measurements for a plasma arc attaching to an aluminium substrate.

\section{INTRODUCTION}

Lightning strikes last for $\mathscr{O}(100) \mathrm{ms}$, producing currents of $\mathscr{O}(10) \mathrm{kA}$ in pulses lasting for $\mathscr{O}(100) \mu \mathrm{s}$. The resulting ionisation generates magnetic fields, coupled to the lightning arc through Joule heating and the Lorentz force, and this injects heat into the plasma, increasing the temperature by $30 \mathrm{kK}$ over the first few microseconds 2 . Over these short time scales, the heat is dissipated through a combination of radiation and convection and, in the case of lightning striking an aircraft, energy is also dissipated by Joule heating within the aircraft material as the electric fields circulate through it.

In the case of lightning attachment to aircraft, there is particular concern for modern designs utilising lightweight composite materials. These materials have much lower thermal and electrical conductivity than traditional materials, such as aluminium, and can undergo delamination and fibre breakage as a result ${ }^{2[3]}$. Numerical models offer a predictive tool which can capture Joule heating and subsequent damage to aircraft substrates, but to accurately capture the behaviour over the early stages of arc attachment, any model implemented relies on accurately modelling the current transferred to the substrate from the arc. Current density is greatest at the centre of the arc, hence any numerical model must be able to predict the plasma dynamics in this region. Due to the high temperatures, radiative heat losses have a significant contribution to the underlying evolution, and thus an accurate radiative model is essential for modelling Joule heating effects in simulating lightning attachment to aircraft substrates. At the centre of the arc, overpressure significantly increases radiative emission which in turn limits further growth of pressure, and temperature, hence an accurate model is essential to avoid

\footnotetext{
a)Electronic mail: ma616@cam.ac.uk
}

over-prediction of these quantities. However, in addition to pressure, radiative emission is also dependent on the composition, temperature, density and geometry of the plasma, and subsequent absorption in the peripheral regions of the arc is also known to be very important to the rate of arc expansion.

A full description of the radiative losses within a plasma arc requires solving for energy balance at every point in the arc, spanning the full range of wavelengths in the electromagnetic radiation. These radiative effects are described in full by the radiative transfer equation (RTE) and this has been solved fully for stationary cylindrically symmetric lightning arcs by Lowke ${ }^{4}$, Lowke and Capriotti ${ }^{5}$ and Hermann and Schade ${ }^{6}$. However for dynamic simulations, the exact solution of the RTE is prohibitively slow and numerical simplifications have been sought ${ }^{7}$. A key challenge here is to balance the accuracy of the method with computational efficiency. Solutions of the RTE, approximate or exact, require knowledge of the spectral properties of the plasma; this information is encapsulated within the monochromatic absorption coefficient and is a measure of the amount of radiation absorbed by the plasma at any given frequency $\sqrt{8}$.

Approximate solutions of the RTE will be collectively referred to in this paper as spectral methods. The simplest approximate solution is the net emission coefficient (NEC) introduced by Lowke ${ }^{9}$, which considers only the radiation emitted by the plasma and, in its simplest form, all self-absorption is neglected. Despite its simplicity it has been found to produce good predictions for the volumetric radiative power in the hottest parts of the plasma, but is less accurate near boundaries or in regions of high pressure $\frac{1011}{13}$. It is implemented by e.g. Chemartin et al! ${ }^{[2}$, Teulet et al.13 and Fusheng et al.14. It has also been applied to modelling lightning streamers by e.g. Da Silva et al! 15 .

More accurate than the NEC are the $\mathrm{P}_{N}$ approximations which were introduced by Lacombe et al. $\frac{16}{16}$ and used by e.g. Aleksandrov $\sqrt{17}$ and Tholin et al. $\frac{18}{}$. They are spherical har- 
monic expansions which satisfy the RTE to $\mathscr{O}(N)$ accuracy. In between these two approaches, the $\mathrm{SP}_{N}$ approximations simplify the complexity of the $\mathrm{P}_{N}$ equations significantly, allowing higher order of accuracy solutions to be calculated for the computational effort. As the radiative emission is wavelength dependent, to reduce the number of calculations, solutions are found for a number of averaged bands in the emission spectrum e.g. 11 in Peyrou et al. ${ }^{19}$, Bartlova et al. ${ }^{20}$ and Tholin et

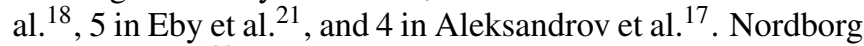
and Iordanidis 22 compare the accuracy of different methods of averaging the absorption coefficient in an $\mathrm{SF}_{6}$ plasma and find that averaged models are sufficiently accurate so long as averages are calculated with a weighting based on their magnitude.

Higher accuracy approximate solutions of the RTE do exist, including the method of partial characteristics introduced by Sevast'yanekd ${ }^{23 \mid 24}$ which tabulates the radiative exchange between volume elements in the plasma in advance by assuming a functional form between each pair of elements. This requires significantly more precalculation than any of the previous methods. Alternatively Monte-Carlo methods, such as those described by Gogel et al. ${ }^{25}$ can be used but these are computationally expensive and impractical for coupling with a full magnetohydrodynamic description 19 .

Within this work, four spectral methods are considered, with their formulation for use in one-dimensional lightning arc simulations evaluated. The NEC, $\mathrm{P}_{1}$ and $\mathrm{SP}_{3}$ are considered, along with an alternative NEC approach considered by Villa et al.26. This latter approach treats the plasma as a grey body of constant emissivity and solves a simplified RTE using the temperature, density and at specific heat capacity of the plasma at a given point. The radiative emission is not dependent on the properties of the neighbouring plasma and hence this method can be thought of as an alternative NEC method 1 . These methods are implemented within a validated magnetohydrodynamics (MHD) code developed by Millmore and Nikiforakis ${ }^{27}$ using an accurate equation of state (EoS)

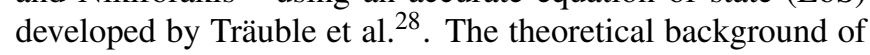
these approaches is detailed in section III, and the results for these one-dimensional implementations given in section III. Based on these results, the implementation of these techniques for efficient multi-dimensional cases is considered. In particular, the NEC, and an improved reduced model, considering an optically dense plasma, are implemented such that the approximate solution to the RTE can be considered a function of the EoS. This allows these approaches to be incorporated in the tabulated data for the equation of state introduced by Träuble et al. Results for these multi-dimensional effects are shown, and validated in section IV, considering a test case of arc attachment to an aluminium substrate. Finally, conclusions and future work are described in section $\mathrm{V}$

\section{MATHEMATICAL MODELS}

\section{A. Plasma evolution equations}

The evolution of a lightning strike is governed by coupling the Euler equations describing the conservation of density $\rho$, momentum $\rho \mathbf{u}$, and energy $E$, with the Maxwell equations governing the electromagnetic interaction of the magnetic field $\mathbf{B}$, and the current density j27/29. The electromagnetic coupling occurs over much faster timescales than the fluid evolution and hence the quasi-static approximation to Maxwell's equations can be used ${ }^{12 \mid 30}$. In this limit, the magnetic field is given by

$$
\mathbf{B}=\nabla \times \mathbf{A}
$$

where $\mathbf{A}$ is the vector magnetic potential. In a material of electrical conductivity $\sigma$, the equations governing the time evolution are

$$
\begin{gathered}
\frac{\partial \rho}{\partial t}+\nabla \cdot(\rho \mathbf{u})=0 \\
\frac{\partial \rho \mathbf{u}}{\partial t}+\nabla \cdot\left(\rho \mathbf{u} \mathbf{u}^{T}+p \mathscr{I}\right)=\mathbf{j} \times \mathbf{B}, \\
\frac{\partial E}{\partial t}+\nabla \cdot(\mathbf{u}(E+p))=\frac{\mathbf{j}^{2}}{\sigma}-S_{R}+\mathbf{u} \cdot(\mathbf{j} \times \mathbf{B}), \\
-\nabla^{2} \mathbf{A}=\mu_{0} \mathbf{j},
\end{gathered}
$$

where $\mu_{0}$ is the permeability of free space. This approach assumes local thermodynamic equilibrium (LTE), assuming that the ionised material can be described with a single local temperature and pressure, which has been demonstrated to be able to accurately predict the behaviour of a lightning plasma ${ }^{31 / 32}$.

To close this system of equations, boundary conditions for current density, a radiative model supplying the source term $S_{R}$, and an EoS describing the interdependence of internal energy $e$, pressure $p$, and temperature $T$ are required. In this work, boundary conditions for current density come from a prescribed current profile at the top of the computational domain, provided by standard wave forms for aerospace testing 33 , and a 19 -species EoS tabulated and validated by ${ }^{28}$ is used. The choice of radiative source term is the focus of this work, but it is worth noting that in addition to closing the system of equations (2)-(5), it can further be used to calculate the frequency-dependent monochromatic absorption coefficient of light, determined by the interaction of photons with the plasma, which is required by the NEC, $\mathrm{P}_{1}$, and $\mathrm{SP}_{N}$ models to calculate the radiation source term.

A full description of the numerical techniques for solving equations 2 to 5 is given in Millmore and Nikiforakis 27? This is a four-step process, solving in the first step the Poisson's equations for the electric potential and magnetic vector potential, and in the second step, solving the evolution equations without forcing terms. In the third step the energy in the plasma is updated to account for radiation and Joule heating, and the final step couples the Lorentz force to the fluid evolution. The general approach to including the radiative source term in the third step is not dependent on the method used to obtain this term, in all cases an ordinary differential equation (ODE) is used to update the energy,

$$
\frac{\partial E}{\partial t}=\frac{\mathbf{j}^{2}}{\sigma}-S_{R} .
$$


For all methods considered, except the grey body approach, the radiative term, $S_{R}$, is computed directly, and then standard ODE solvers are used to update the energy. For the grey body model, the solution to the ODE is coupled to the approximate solution to the RTE, this method is described in section II B.

\section{B. Grey body model}

The grey body model is of simplified NEC model used by Villa et al.26. Although this approach simplifies the underlying model for solving the RTE, by neglecting spectral and geometric dependencies, it does add complexity in the techniques for solving equation (6); this process can be broken down into three steps. In the first, the plasma is assumed to absorb energy from its surroundings which are at an ambient temperature, $T_{0}$. For a grey body of emissivity $\kappa_{\text {emiss }}$ the energy absorbed is given by $\kappa_{\text {emiss }} \sigma_{S B} T_{0}^{4}$ where $\sigma_{S B}$ is the Stefan-Boltzmann constant and Villa et al. use $\kappa_{\text {emiss }}=60.0$. This absorption is treated as a source term alongside the Joule heating, and the energy is updated using the first-order explicit step,

$$
E^{n+1 / 2}=E^{n}+\Delta t\left(\frac{\mathbf{j}^{2}}{\sigma}+\kappa_{\text {emiss }} \sigma_{S B} T_{0}^{4}\right)
$$

where $\Delta t$ is the discretised time step of the simulation.

In the second step an intermediate temperature, $T^{n+1 / 2}$, corresponding to this updated energy, is calculated, and from this temperature, and the EoS, mass fractions of the chemical species are obtained. From these the species-averaged heat capacity and heat of formation heats must be determined; the specific heat capacity at constant volume, $c_{v}^{i}$, for a diatomic species of molar mass $M_{k}$ depends on the specific gas constant $R_{k}=\frac{\hat{R}}{M_{k}}$ and the temperature $T$,

$$
c_{v}^{i}(T)=\left(\frac{5}{2}+\left(\frac{T_{M}}{T}\right)^{2} \frac{\exp \left(T_{M} / T\right)}{\left(\exp \left(T_{M} / T\right)-1\right)^{2}}\right) R_{k},
$$

where $T_{M}$ is a characteristic temperature for the atomic species involved. For a monatomic species, $c_{v}^{i}$ is given by:

$$
c_{v}^{i}(T)=\frac{3}{2} R_{k}
$$

The specific heats of formation for each species, $\Delta h_{f}^{0, i}$, are taken from thermochemical tables $34 \sqrt[36]{3}$. The species-averaged values are then calculated from these quantities, and the mass fractions, $x_{i}$, as provided by the EoS, according to

$$
c_{v}=\sum_{\text {species }} c_{v}^{i}\left(T^{n+1 / 2}\right) x_{i}
$$

and

$$
\Delta h_{f}^{0}=\sum_{\text {species }}\left(\Delta h_{f}^{0}\right)^{i} x_{i}
$$

From these, the specific heat capacity at constant pressure, $c_{p}$, as well as the corresponding temperature associated with the heat increase having taken place at constant pressure, $T_{b}$, can be calculated:

$$
c_{p}=\alpha c_{v}
$$

and

$$
T_{b}=\alpha T^{n+1 / 2}
$$

where

$$
\alpha=\frac{\left(e-\Delta h_{0}^{f}\right)}{c_{v} T^{n+1 / 2}} .
$$

The final step calculates the temperature of the plasma after radiative emission. Again the plasma is modelled as a grey body, and emission is described by

$$
\rho c_{p} \frac{d T}{d t}=-\sigma_{S B} \kappa_{e m i s s} T^{4}
$$

This simplified RTE is solved analytically by rearranging and integrating from time $t$ to $t+\Delta t$, to obtain an updated temperature, $T_{n b}$. This produces an explicit expression for $T_{n b}$,

$$
T_{n b}=\left(T_{b}^{-3}+\frac{3 \sigma_{S B} \kappa_{\text {emiss }} \Delta t}{\rho c_{p}}\right)^{-1 / 3},
$$

and from this, the total energy at temperature $T_{n b}$ is found by adding the enthalpic, thermal and kinetic contributions,

$$
E^{n+1}=\rho^{n+1}\left(\Delta h_{0}^{f}+c_{p} T_{n b}+\frac{1}{2}\left(u^{n+1}\right)^{2}\right)
$$

\section{Net emission coefficient}

The NEC improves upon the grey body approach by considering the spectral dependencies of the radiative emission. The radiation intensity at a given frequency per unit solid angle, $I_{v}$, is calculated by solving the RTE which, for spherical symmetry in a non-scattering plasma with unit refractive index, is:

$$
\frac{\partial I_{v}}{\partial r}=\kappa_{v}^{\prime} B_{v}-\kappa_{v}^{\prime} I_{v}
$$

where $\kappa_{v}^{\prime}(T, p)$ is the modified absorption coefficient taking into account stimulated emission, 37

$$
\kappa_{v}^{\prime}=\kappa_{v}\left[1-\exp \left(-\frac{h v}{k_{B} T}\right)\right],
$$

with $h$ and $k_{B}$ being the Planck and Boltzmann constants respectively, and the spectral radiation intensity $B_{v}$ is given by Planck's law for black body radiation,

$$
B_{v}=\frac{2 h v^{3}}{c^{2}\left[\exp \left(\frac{h v}{k_{B} T}\right)-1\right]},
$$


where $c$ is the speed of light. Under the assumption of LTE, Kirchoff's law holds, hence the emission coefficient at frequency $v, \varepsilon_{v}$, satisfies

$$
\frac{\varepsilon_{v}}{\kappa_{v}^{\prime}}=B_{v} .
$$

The NEC, $\varepsilon_{N}$, is then given by integrating over all frequencies for a plasma contained within an isothermal sphere of radius $R_{P}$

$$
\varepsilon_{N}\left(T, p, R_{p}\right)=\int_{0}^{\infty} B_{v} \kappa_{v}^{\prime} \exp \left(-\kappa_{v}^{\prime} R_{P}\right) d v
$$

where $R_{p}$ is the decay length of the plasma. An assumption of $R_{P}=0$ is equivalent to assuming an optically thin plasma for which about $90 \%$ of the radiation is absorbed. Within this paper, $R_{p}=0$ is referred to as the NEC model, and non-zero values of $R_{p}$ are reduced NEC models. In order to obtain the radiative source term from this value, the NEC is related to the divergence of radiative flux $\mathbf{q}$ and hence $S_{R}$ by 4

$$
S_{R}=\nabla \cdot \mathbf{q}=4 \pi \varepsilon_{N}
$$

Computationally, computing the integral in equation (22) requires a discretisation of the frequency, $v$. Following Nordborg and Indianis 22 , the absorption coefficient is computed using band averaged values of $\kappa_{v}$ and $B_{v} \kappa_{v}$, denoted $\bar{\kappa}_{i}$ and $(\overline{B \kappa})_{i}$ respectively, and a Planck-average is used to compute these values, for example

$$
\bar{\kappa}_{i}=\frac{1}{\int_{\Delta v_{i}} B_{v} d v} \int_{\Delta v} B_{v} \kappa_{v} d v,
$$

where the frequency range is given by $\Delta v$. It is sufficient for five band-averaged values to be used to compute $\varepsilon_{N}$, hence the radiative source term is given by

$$
S_{R}=4 \pi \sum_{i=1}^{5}(\overline{B \kappa})_{i} \exp \left(-\bar{\kappa}_{i} R_{p}\right)
$$

Further details for computing these quantities are given in appendix A.

For the NEC and reduced NEC models, further computational gains are available since the NEC itself only depends on local pressure and temperature. These quantities can then be computed as part of the tabulated EoS and interpolated values used to compute radiative losses.

\section{D. $\mathbf{P}_{1}$ approximation}

The $\mathrm{P}_{N}$ approximations improve upon the NEC by beginning to account for geometric effects which lead to selfabsorption of radiation by the plasma. The derivation of the $\mathrm{P}_{N}$ approximations starts from the directional RTE, neglecting scattering in the direction $\hat{\mathrm{s}}^{21}$,

$$
\hat{\mathbf{s}} \cdot \nabla I_{V}=\kappa_{v}\left(B_{V}-I_{V}\right) .
$$

The radiation intensity is decomposed into directiondependent coefficients of the spherical harmonics $Y_{l}^{m}(\hat{\mathbf{s}})$ and spatial-dependent coefficients $P_{l}^{m}(\mathbf{r})$ :

$$
I_{v}(\mathbf{r}, \mathbf{s})=\sum_{l=0}^{\infty} \sum_{m=-l}^{l} P_{l}^{m}(\mathbf{r}) Y_{l}^{m}(\hat{\mathbf{s}}) .
$$

Due to the complexities of evaluating this expression for higher-order approximations, in this work only the $P_{1}$ approximation is used, for which only the first term in the expansion is kept. Eby et $\mathrm{al}^{21}$ show that this is of the form

$$
I_{v}(\mathbf{r}, \mathbf{s})=\frac{1}{4 \pi}\left(G_{v}+3 \mathbf{q}_{v} \cdot \hat{\mathbf{s}}\right) .
$$

where $G_{V}$ is the incident radiation defined by integrating over solid angle $\Omega$,

$$
G_{V}=\int_{4 \pi} I_{V}(\mathbf{r}, \hat{\mathbf{s}}) d \Omega
$$

and $\mathbf{q}_{v}$ is the radiative flux,

$$
\mathbf{q}_{v}=\int_{4 \pi} I_{V}(\mathbf{r}, \hat{\mathbf{s}}) \hat{\mathbf{s}} d \Omega
$$

Substitution of equation (28) into equation (26) generates the governing equations,

$$
\nabla G_{V}=-3 \kappa_{v} \mathbf{q}_{v}
$$

and

$$
\nabla \cdot \mathbf{q}_{v}=\kappa_{v}\left(4 \pi B_{v}-G_{v}\right)
$$

These can be combined into a single Helmholtz-type equation for $G_{V}$,

$$
\nabla \cdot\left(\frac{1}{\kappa_{v}} \nabla G_{v}\right)=3 \kappa_{v}\left(G_{v}-4 \pi B_{v}\right) .
$$

As with the NEC approach, five frequency bands are used for solving this equation, and the volumetric radiation source term is given by

$$
S_{R}=\nabla \cdot \overline{\mathbf{q}}=\sum_{i=1}^{5} 4 \pi(\kappa \bar{B})_{i}-\bar{\kappa}_{i} \bar{G}_{i}
$$

Unlike the NEC approach, the geometric dependencies of equation (34) mean that the radiative losses cannot be included within the EoS data. In this work, the solution to this equation uses a five-point stencil, to produce a block tridiagonal system, and is achieved through the ADI method 38 .

\section{E. $\mathrm{SP}_{3}$ Approximation}

To obtain higher order $\mathrm{P}_{N}$ approximations the complexity and number of equations increases. The $\mathrm{SP}_{N}$ approximations are a way of simplifying these equations and reducing them in 
number for the case of an optically thick plasma with length scale $x_{\text {ref }}$ and characteristic opacity $\kappa_{\text {ref }}$ such that

$$
\varepsilon=\frac{1}{\kappa_{r e f} x_{r e f}}
$$

satisfies $0<\varepsilon \ll 1$. Assuming that thermal diffusion occurs with thermal conductivity $k_{T}$, the energy balance equation can be written

$$
\rho c_{p} \frac{\partial T}{\partial t}=\nabla \cdot\left(k_{T} \nabla T\right)-\int_{v_{1}}^{\infty} \int_{\mathscr{S}^{2}} \kappa_{v}\left(B_{v}-I_{v}\right) d \Omega d v
$$

and the directional RTE 26 can be rescaled ${ }^{39}$, giving

$$
\varepsilon^{2} \frac{\partial T}{\partial t}=\varepsilon^{2} \nabla \cdot\left(k_{T} \nabla T\right)-\int_{v_{1}}^{\infty} \int_{\mathscr{S}^{2}} \kappa_{v}\left(B_{v}-I_{\nu}\right) d \Omega d v
$$

and

$$
\forall v>v_{1}, \quad \Omega \in \mathscr{S}^{2}: \varepsilon \Omega \cdot \nabla I=\kappa_{v}\left(B_{v}-I_{v}\right) .
$$

This latter equation can be rearranged to give

$$
I_{V}=\left(1-\frac{\varepsilon}{\kappa_{v}} \Omega \cdot \nabla\right)^{-1} B_{V}
$$

and then expanded in powers of $\frac{\varepsilon}{\kappa_{v}} \Omega \cdot \nabla$. By introducing the integral of the radiation intensity at given freqency over solid angles,

$$
\psi=\int_{\mathscr{S}^{2}} I_{V} d \Omega
$$

retaining terms up to $\mathscr{O}\left(\varepsilon^{8}\right)$, substituting into equation (37), and simplifying, two expressions are obtained, 39

$$
\begin{aligned}
& -\nabla \cdot\left(\frac{\mu_{1}}{\kappa_{v}} \nabla \psi_{1, v}\right)+\kappa_{v} \psi_{1, v}=\left(4 \pi B_{v}\right) \kappa_{v} \\
& -\nabla \cdot\left(\frac{\mu_{2}}{\kappa_{v}} \nabla \psi_{2, v}\right)+\kappa_{v} \psi_{2, v}=\left(4 \pi B_{v}\right) \kappa_{v}
\end{aligned}
$$

where $7 \mu_{1,2}=3 \mp \sqrt{6 / 5}$. In this model the following boundary conditions were assumed 21 .

- A symmetry condition: $\left.\partial_{r} G_{V}\right|_{r=0}=0$,

- No flux at the air boundaries: $\mathbf{n} \cdot \nabla G_{V}=0$

- No radiation transfer to the solid surfaces: $\mathbf{n} \cdot \nabla G_{v}=0$

These boundary conditions decouple equations (41) and (42), which can then be treated as Helmholtz equations, and are again discretised using the ADI method with 5 bands used for the frequency averaging of these equations. For the $\mathrm{SP}_{3}$ approximation, this then requires 10 Helmholtz equations to be solved each time step. The radiative power for the $\mathrm{SP}_{3}$ approximation is given by

$$
S_{R}=\int_{0}^{\infty} \nabla \cdot\left(\frac{1}{\kappa_{v}} \nabla\left(a_{1} \psi_{1, v}+a_{2} \psi_{2, v}\right)\right) d v
$$

where $30 a_{1,2}=(5 \mp 3 \sqrt{5 / 6}), \frac{19}{19}$ and again, this quantity is approximated by a sum over the band-averaged values,

$$
S_{R}=\sum_{i=1}^{5} \frac{a_{1}}{\mu_{1}}\left(\bar{\kappa} \psi_{1, i}-4 \pi \bar{\kappa} B_{i}\right)+\frac{a_{2}}{\mu_{2}}\left(\bar{\kappa} \psi_{2, i}-4 \pi \bar{\kappa} B_{i}\right)
$$

Again, the geometric dependency of the $\mathrm{SP}_{3}$ method means that these values cannot be tabulated as part of the EoS data.

\section{NUMERICAL RESULTS}

In order to compare the different radiative models, an initial assessment was made using a one-dimensional, cylindrical plasma arc. This follows experimental and numerical studies of Villa et al. ${ }^{26}$, and was used to validate the underlying numerical framework by Millmore and Nikiforakis 27. Experimentally, a plasma arc attaches to a highly conductive metal substrate within an set up large enough such that the arc can be treated as cylindrical. The arc is driven by an input current from an electrode with a profile given by

$$
I=I_{0} \exp (-\alpha t) \sin (\beta t)
$$

The simulation is initialised with a high-temperature preheated arc region to approximate the initial breakdown of air generating this structure. This approach avoids the need to incorporate the complex physics of the breakdown itself, and has been shown by Tholin et al ${ }^{18}$ not to affect the overall evolution of the arc. The initial conditions for this test case are given in table I] and represent a preheated arc of radius $2 \mathrm{~cm}$ with a Gaussian temperature profile and maximum temperature $8680 \mathrm{~K}$. The simulation was implemented in the domain $x \in[0,25] \mathrm{cm}$ and, following the method of Villa et al, a current density profile of the form $\mathbf{J}=J(\mathbf{r}) \mathbf{e}_{z}$ was used. The remaining initial conditions are given in table $\mathbb{I}$.

\begin{tabular}{|c|c|c|}
\hline & $r<0.02$ & $r>0.02$ \\
\hline$\rho\left(\mathrm{kgm}^{-3}\right)$ & 0.9 & 1.225 \\
\hline$u_{r}\left(\mathrm{~ms}^{-1}\right)$ & 100 & 0 \\
\hline$E(\mathrm{~J})$ & $20 \times 335014 \exp \left(-\frac{x^{2}}{0.5^{2}}\right)$ & 335014 \\
\hline
\end{tabular}

TABLE I: Initial conditions for a cylindrical plasma arc, reproducing the experiment of Villa et al.26.

Figures 1 and 2 respectively show the pressure and temperature evolution at three output times for the grey body, NEC, $\mathrm{P}_{1}$ and $\mathrm{SP}_{3}$ models. In all cases, the pressure profiles show a leading shock wave propagating radially outwards, followed by a strong rarefaction. This results in a pressure decrease at the centre of the arc, though due to the oscillatory current input, this is not a monotonic decrease. Due to lower density at the centre of the arc, the temperature remains at its highest in this region, although this again decreases over time, as the input current reduces.

For all radiative models, the behaviour of the shock wave is largely unchanged. Due to the lower temperatures, the material evolution and the equation of state dominate the behaviour 


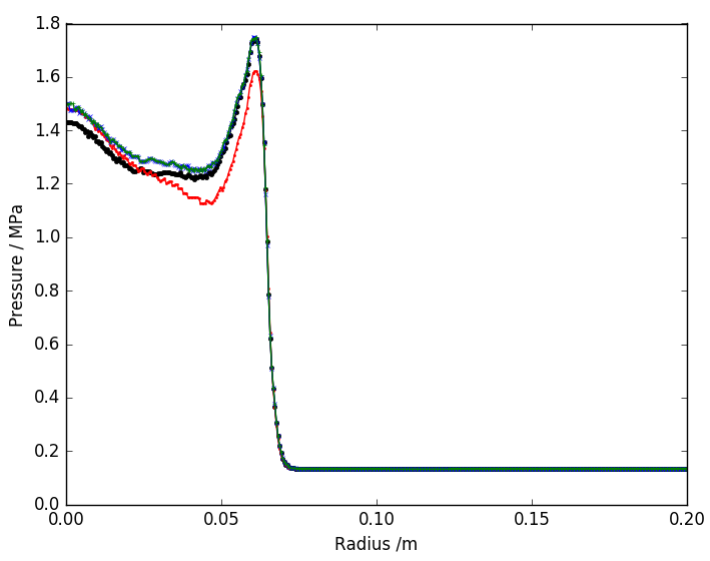

(a) $20 \mu \mathrm{s}$

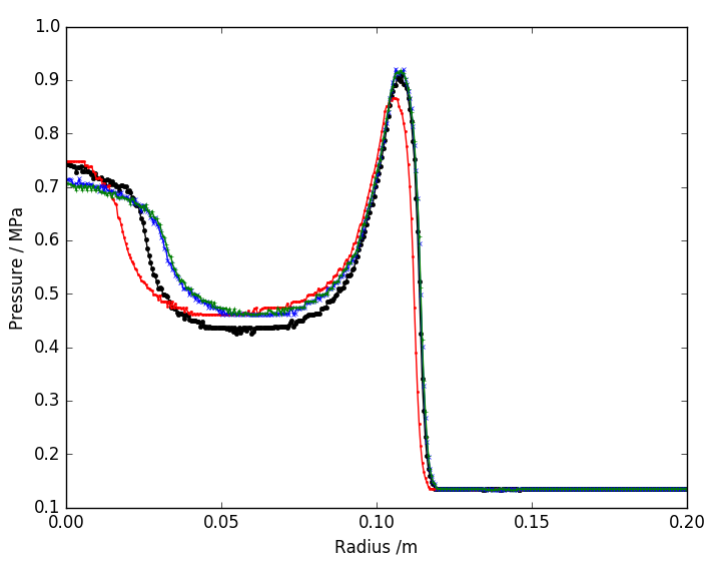

(b) $60 \mu \mathrm{s}$

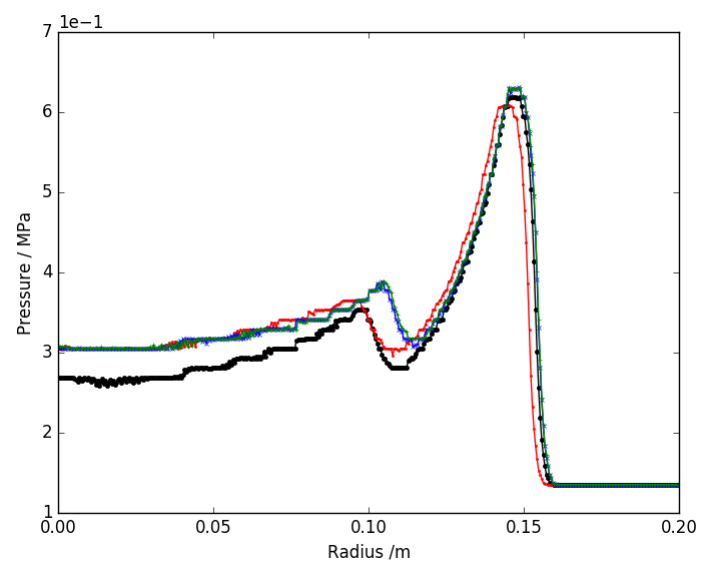

(c) $100 \mu \mathrm{s}$

FIG. 1: Pressure evolution of a cylindrical plasma arc modelled with four different radiative source terms. All four approaches show qualitatively the same behaviour, especially for the leading shock wave, which is governed primarily by

the equation of state. Towards the centre of the arc, differences between the methods are visible; the NEC approach initially predicts a lower pressure, whilst at late times, the grey body approach has a lower pressure than the three spectral methods.

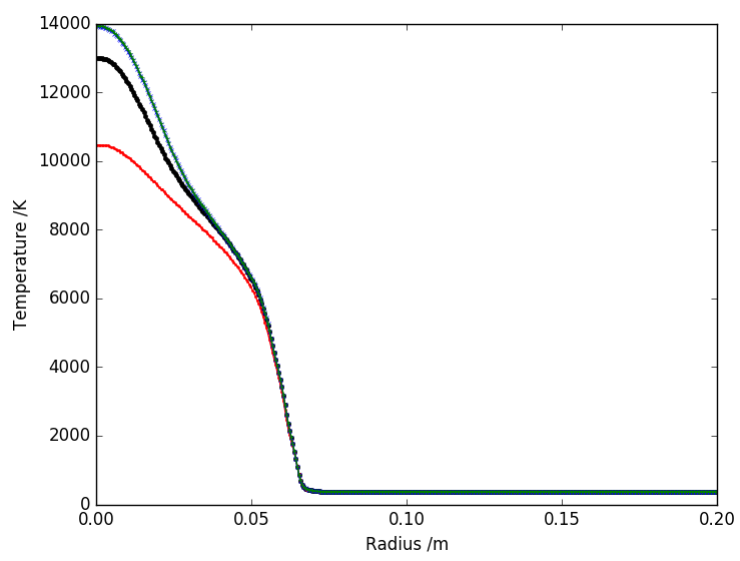

(a) $20 \mu \mathrm{s}$

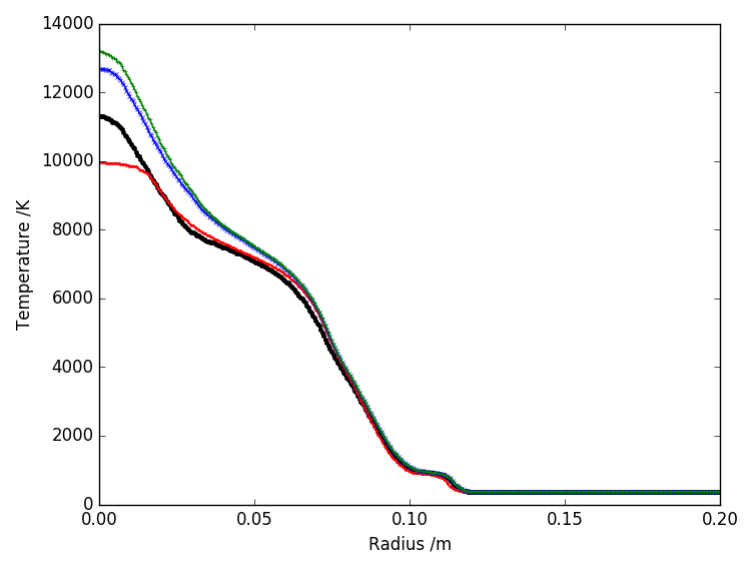

(b) $60 \mu \mathrm{s}$

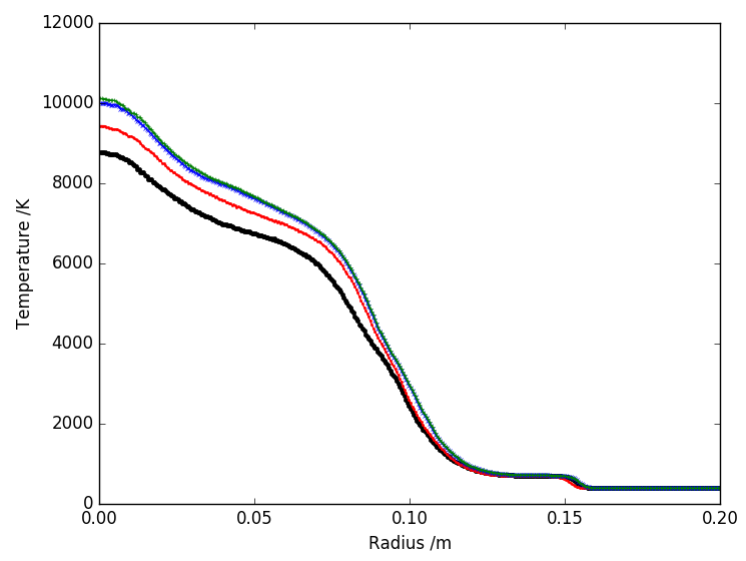

(c) $100 \mu \mathrm{s}$

FIG. 2: Temperature evolution of a cylindrical plasma arc modelled with four different radiative source terms. The NEC approach consistently produces lower temperatures than the $\mathrm{P}_{1}$ and $\mathrm{SP}_{3}$ methods, though these profiles are comparable at late times. The grey body approach is initially similar to the $\mathrm{P}_{1}$ and $\mathrm{SP}_{3}$ methods, but from around $20 \mu \mathrm{s}$ onwards predicts a lower temperature. 
here, as demonstrated by Träuble et al ${ }^{28}$. However, as is visible in the pressure profile in figure 1, even at early times, after $10 \mu \mathrm{s}$, radiative effects are clear at the centre of the arc. At this time, the NEC approach results in a lower central pressure, around $0.5 \mathrm{MPa}$ less than the other three models. At later times, however, the three spectral methods result in comparable pressure profiles, whilst the grey body approach leads to a lower pressure, $25 \%$ lower after $150 \mu \mathrm{s}$. When comparing the three spectral methods, the differences between the pressure profiles at early times is a direct effect of geometric effects. The $\mathrm{P}_{1}$ and $\mathrm{SP}_{3}$ take local gradients into account in solving for the radiative source term; at early times, when the arc radius is small, these effects are a have a more significant contribution. At later times, the local arc properties dominate, and all three spectral methods have similar behaviour. By comparison, the simplicity of the grey body approach shows that differences in the arc behaviour develop as a result of the choice of radiative term.

Similar effects are visible in the temperature profile, shown in figure 2. In this case, the grey body approach is again comparable to the $\mathrm{P}_{1}$ and $\mathrm{SP}_{3}$ methods at early times, and results in an lower temperature value at the arc centre at later times. Similarly, the initial low prediction of the NEC approach is visible, though in this case, the temperature predicted by the NEC model remains lower throughout the evolution of the arc, though at late times it is closer to the other two spectral methods than the grey body approach.

\section{IMPLEMENTATION AND VALIDATION WITHIN A MULTIPHYSICS FRAMEWORK}

This one-dimensional study suggests that the spectral methods could offer an improvement in accuracy in modelling the centre of a lightning arc. To further confirm this, additional validation is required; in particular a comparison to the behaviour at the centre of the arc. The results in section III show that the behaviour of the leading shock wave and the arc expansion are largely unaffected by the choice of radiative model. Since these are the easiest characteristics of an arc evolution to measure experimentally, these offer excellent validation material for the underlying system of equations and equation of state. In order to extend this validation to include the radiative model, conditions at the arc centre are required. Such data has been recorded by Martins ${ }^{1}$, for which the thermodynamic properties of the arc can be inferred from the optical intensity. In particular, Martins considers an arc attachment to an aluminium substrate, the modelling of which requires the axisymmetric multiphysics approach introduced in Millmore and Nikiforakis 27 .

When considering the three spectral methods introduced in section $\left[\mathrm{II}\right.$, the geometric dependence of the $\mathrm{P}_{1}$ and $\mathrm{SP}_{3}$ approaches requires the solution of multiple Helmholtz equations every time step. In more than one dimension, these solutions become computationally prohibitive, hence these models are not used within this section. However, the performance of the NEC approach at later times suggests this may offer improved accuracy in modelling the conditions at the centre of the arc. Additionally, the optically thin assumption of the NEC can be removed by considering a decay length, as described in section IIC In this work, a decay length of $R_{p}=2 \mathrm{~mm}$ was used Since the approximate solutions to the NEC and the reduced NEC can be tabulated based on material properties, and included with the equation of state, these approaches remain efficient in multiple dimensions.

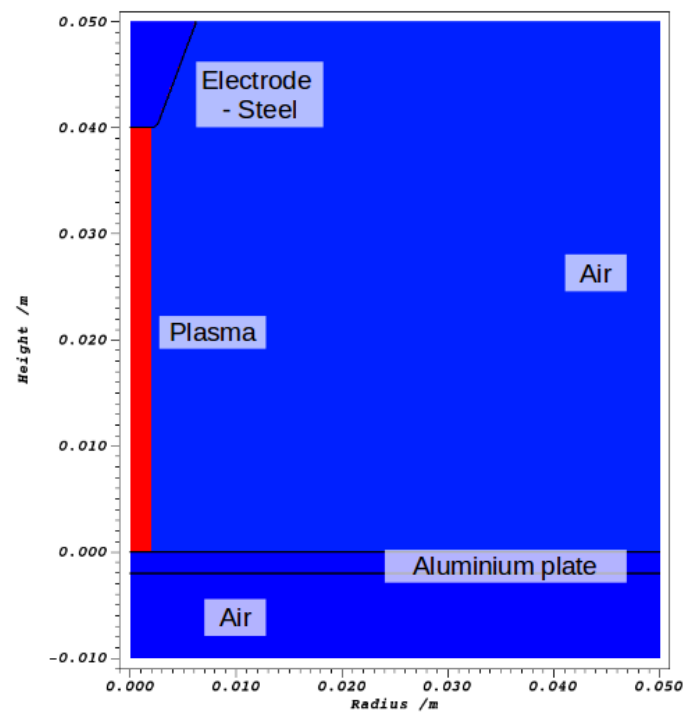

FIG. 3: Initial geometry used in the multimaterial code, with the location of the pre-heated arc shown.

In order to compare to the experimental results of Martins 1 , the configuration shown in Figure 3 is used. Within this model, there is a geometric boundary at $r=0$, and for the thermodynamic variables, all other boundaries are transmissive, zero-gradient Neumann boundaries. For magnetic potential, the normal component to each boundary has is a zerogradient Neumann condition, whilst the transverse component vanishes. For the electric potential, all boundaries are zerogradient Neumann conditions except for the outer edge of the aluminium plate, which is grounded $(\phi=0)$ and the upper edge of the electrode, which has an input current condition.

The current input used by Martins is one of the ARP standard input current profiles 33 , which has an analytical form

$$
I(t)=I_{0}\left(e^{-\alpha t}-e^{-\beta t}\right)\left(1-e^{-\gamma t}\right)^{2} .
$$

where the coefficients are given in table $I$. The remaining ini-

\begin{tabular}{|c|c|c|c|}
\hline$I_{0}(\mathrm{~A})$ & $\alpha\left(\mathrm{s}^{-1}\right)$ & $\beta$ & $\gamma$ \\
\hline 192039 & 32377 & 144719 & 2667492 \\
\hline
\end{tabular}

TABLE II: Fitted values for the current profile described by equation (46) as used in the experiment of Martins 1 .

tial conditions for these tests are given in table III

In order to obtain thermodynamic behaviour from the optical intensity of the plasma arc, an assumption as to the mechanism for the generation of this emission must be made. To 


\begin{tabular}{|c|c|c|c|}
\hline Material & $\rho\left(\mathrm{kgm}^{-3}\right)$ & $\mathbf{u ~}\left(\mathrm{ms}^{-1}\right)$ & $p(\mathrm{~Pa})$ \\
\hline Air $(z<-0.002 \mathrm{~m})$ & 1.225 & $\mathbf{0}$ & 94108 \\
\hline Plate $(-0.002<z<0 \mathrm{~m})$ & 2710 & $\mathbf{0}$ & 94108 \\
\hline Plasma $(r<0.002 \mathrm{~m})$ & 1.225 & $\mathbf{0}$ & $4.02 \times 10^{6}$ \\
\hline Plasma $(r>0.002 \mathrm{~m})$ & 1.225 & $\mathbf{0}$ & 94108 \\
\hline
\end{tabular}

TABLE III: Initial conditions for the attachment of a plasma arc to an aluminium substrate for reproducing the experiment of Martins $\frac{1}{1}$.

achieve this, Martins ${ }^{1}$ develops a NEC for an arc comprising nitrogen and oxygen, for which the singly ionised states, $\mathrm{N}^{+}$and $\mathrm{O}^{+}$, are the only radiating species. Contributions to the absorption coefficient are based on emission spectra for the bound-bound contributions, but use a linear relationship for the bound-free and free-free contributions. Self absorption is treated using a temperature- and pressure-dependent shell model. Together with the measured radiation intensity of the arc, this NEC allows arc temperature and electron number density to be inferred; Martins states measurement error of 5\% and $12 \%$ respectively. Pressure estimates are also available, though require additional use of an equation of state, with errors between $20 \%$ at early times (first few microseconds) up to $36 \%$ at later times.

In order to compare results from the model presented within this work, the temperature is used, since the error measurements on this quantity are smallest. It is noted that the profiles shown by Martins do not show a decay at larger radii, in contrast to other experimental results by $\mathrm{Hsu}^{41}$ and other simulation results, e.g. Chemartin et al.$^{42}$ and Millmore and Nikiforakis 27 . The reason for this is unknown; in order to make a consistent comparison, the central arc temperature is used. Results for this central temperature are shown in table [V] For the grey body approach, the arc temperature is consistently higher than that measured experimentally, in contrast to the results shown in section III] However, the arc itself is at a higher temperature, and this will alter the spectral dependencies of the radiative emission, which are not modelled under this approach, which may account for this change in behaviour. The NEC for an optically thin plasma consistently under-predicts temperature, as it did for the results shown in section III] In this case, the higher temperatures within the arc mean that the optically thin assumption is likely to be even less valid than for the arc shown in figure 2. However, by introducing an absorption length into the NEC approach, the reduced NEC gives temperature much closer to those of Martins. Although errors are slightly larger than those reported by Martins, the fact that both measurements are based on underlying physical models suggest that this approach is performing well. In particular, a reduced version of the NEC approach, which can be tabulated as part of an equation of state, can improve the accuracy of plasma arc temperatures without excessive computational cost.

Figure 4 shows the temperature and pressure profiles of the arc attachment to aluminium for the three radiative models. In each case, the respective differences in temperature within the arc, as described in table IV, are clear within the images. The lower temperature of the NEC approach results in a simi- lar lower pressure within the arc, whilst for the reduced NEC pressure is slightly higher at the centre of the arc. It is believed that despite the lower central temperatures for the reduced NEC, compared with the grey body, lower radiative emission drives less material from the arc centre, reducing energy losses and allowing higher pressure to be maintained. Despite these differences, in all cases, the actual arc profile is qualitatively unchanged, as would be expected since the bulk evolution is dependent on the equation of state, as shown in section III.

Although the arc behaviour is not affected by the choice of radiative model, the attachment point to the substrate is at the centre of the arc. Here, the varying conditions may then have a direct effect on the loading and damage to the substrate. In order to investigate the effect this may have on the substrate properties, figure 5 shows the pressure (stress) profile directly beneath the surface of the substrate. For all three models, similar qualitative behaviour is observed; an initial high-pressure loading generates two features, an outwards moving wave, and a subsequent, higher pressure region which remains largely stationary. The outwards moving wave is connected to the pressure loading of the shock wave, whilst the conditions of the arc at the attachment point result in a continuous loading at the centre. At later times, interactions within the substrate, and between the substrate and the arc lead to a rarefaction (expansion) within the aluminium, hence negative pressures are experienced as the material is placed under tension. There are clear differences between the three radiative models, which largely coincide with the temperature behaviour at the centre of the arc. The outwards-moving wave moves fastest for the grey body approach, and slowest for the NEC, and a similar pattern is observed for the central loading. For this latter case, however, at late times, the grey body approach and the reduced NEC demonstrate similar behaviour, in both cases with a higher magnitude that the NEC approach. It is noted that for aluminium, these conditions, coupled with the high electrical conductivity, and hence low Joule heating effects within the substrate, mean that temperature does not rise more than a couple of Kelvin over this time scale.

It is clear that the radiative model does have an effect on the evolution of the loading on the substrate. Quantifying this behaviour requires a material damage model, and is beyond the scope of the current work, which serves to highlight this possibility. For aluminium, it is reasonable to assume that the high electrical and thermal conductivity of the substrate mean that these differences in loading will have a small impact on the resultant damage behaviour. However, for composite materials, these effects could be more significant. Under lightning strike, these materials can suffer delamination, and this pro- 


\begin{tabular}{|c|c|cc|cc|cc|}
\hline & \multicolumn{7}{|c|}{ Central Temperature (kK) } \\
\hline Times $(\mu \mathrm{s})$ & Martins & Grey Body & (error) & NEC & (error) & Reduced NEC & (error) \\
\hline 2 & 37.4 & 48.1 & $(28 \%)$ & 18.5 & $(-50 \%)$ & 39.9 & $(6.7 \%)$ \\
4 & 34.8 & 54.8 & $(57 \%)$ & 13.4 & $(-61 \%)$ & 36.6 & $(5.1 \%)$ \\
6 & 32.8 & 49.5 & $(50 \%)$ & 12.5 & $(-62 \%)$ & 30.6 & $(-6.7 \%)$ \\
9 & 28.6 & 42.7 & $(49 \%)$ & $12.1(-58 \%)$ & 25.5 & $(-11 \%)$ \\
14 & 25.4 & 36.9 & $(45 \%)$ & $12.0(-53 \%)$ & 22.4 & $(-12 \%)$ \\
20 & 22.9 & 32.5 & $(42 \%)$ & $11.9(-48 \%)$ & 20.9 & $(-8.7 \%)$ \\
26 & 20.7 & 29.9 & $(44 \%)$ & $11.8(-43 \%)$ & 21.0 & $(1.4 \%)$ \\
36 & 19.1 & 25.4 & $(25 \%)$ & $11.8(-29 \%)$ & 21.5 & $(9.4 \%)$ \\
\hline
\end{tabular}

TABLE IV: Central arc temperatures, and errors, determined experimentally by Martins can be compared to those predicted by the grey body, NEC and reduced NEC models. The grey body approach consistently over predicts temperature, whilst the NEC consistently under predicts temperature. The reduced NEC shows a much closer fit than the other two models.

cess starts within around $10 \mu s^{43}$. As a result, the differences in pressure loading predicted by the radiative model may have a direct implication for understanding material damage.

\section{CONCLUSIONS}

The aim of this work was to investigate the effects of the choice of model for quantifying the radiative losses at the centre of a plasma arc, within a multiphysics magnetohydrodynamics code. Both the behaviour of the arc in isolation, and when attaching to a substrate, was considered, as was the evolution within the substrate itself. Four radiative models were considered, all dependent on the plasma composition, as determined by the equation of state. The simplest of these, the grey body approach, uses uniform absorption properties and can be computed from the bulk thermodynamic properties of the plasma, and was initially used by Villa et al. ${ }^{26}$ The NEC approach, which can either assume an optically thin plasma, or a reduced approach with a finite decay length for radiative emission, is dependent on the full plasma composition, as determined by the equation of state, and the associated spectral absorption properties. Two further approaches, the $\mathrm{P}_{1}$ and $\mathrm{SP}_{3}$ approximations, take the geometric behaviour of the arc into account, in addition to local properties. These latter three approaches, referred to as spectral methods, use a composition-dependent absorption coefficient, calculated following the work of Chauveau et al..$^{7}$.

Two key questions investigated in this work were whether the radiative model affected the accuracy of the simulation of a plasma arc, and whether the effects of arc attachment to a substrate were altered by the model. Additionally, an initial assessment into the computational efficiency of the approaches was considered. This considered a one-dimensional test case, as used by Villa et al ${ }^{26}$ for both experimental an numerical studies. The results show that the three spectral methods generally gave similar results, and these differed noticeably from the grey body approach. The difference between the $\mathrm{P}_{1}$ and $\mathrm{SP}_{3}$ was particularly small, though at early times, the NEC approach tended to predict lower temperatures and pressures.

In order to compare the results from the different radiative models to experimental data, an experiment considering an arc attachment to aluminium, conducted by Martins ${ }^{1}$ was considered. This could be approximated by two-dimensional axisymmetry, and this introduced additional geometric complexity for computing the $\mathrm{P}_{1}$ and $\mathrm{SP}_{3}$ approximations; in practice, these were computationally inefficient. However, the similarities between the NEC and the $\mathrm{P}_{1}$ and $\mathrm{SP}_{3}$, and the fact that it could be implemented as part of the plasma equation of state, and did not require additional computation during a simulation, suggested that this method could be used in the comparison to the experimental results. Additionally, the initial assumptions of an optically thin plasma do not necessarily hold at the centre of a plasma arc, hence the reduced NEC was also investigated at this point, with a finite decay length for radiative emission. Obtaining the properties of a plasma arc experimentally is challenging, and requires additional modelling for inference from optical emission, but it was shown that the reduced NEC provided a reasonable description of the thermodynamics of the arc centre, performing better than the grey body and original NEC approaches.

The effects of the radiative model on the coupling between the arc and an aluminium substrate was then considered. This investigated the practical application of understanding how the non-linear multiphysics simulation of both the arc and the substrate can help understand damage as a result of lightning strike. Here it was shown that pressure within the substrate over the first $\sim 30 \mu$ s was dependent on the radiative model, with the grey body approach resulting in faster pressure evolution than the spectral approaches, and the optically thin NEC causing slower pressure evolution. This has potential implications for modelling damage to low conductivity composite substrates, where it is found that delamination of the substrate has started within these time scales.

This work has highlighted the importance of the choice of radiative model for accurate simulation of lightning attachment and damage. However, it has been shown that complex models solving for geometric behaviour within the arc, which require the solution to multiple Helmholtz equations within a single simulation time step, do not provide a substantial benefit over NEC-based approaches. Future work based on this could improve the radiative model further based on the NEC approach, for example improving the reduced NEC model with a decay length based on local plasma properties. 


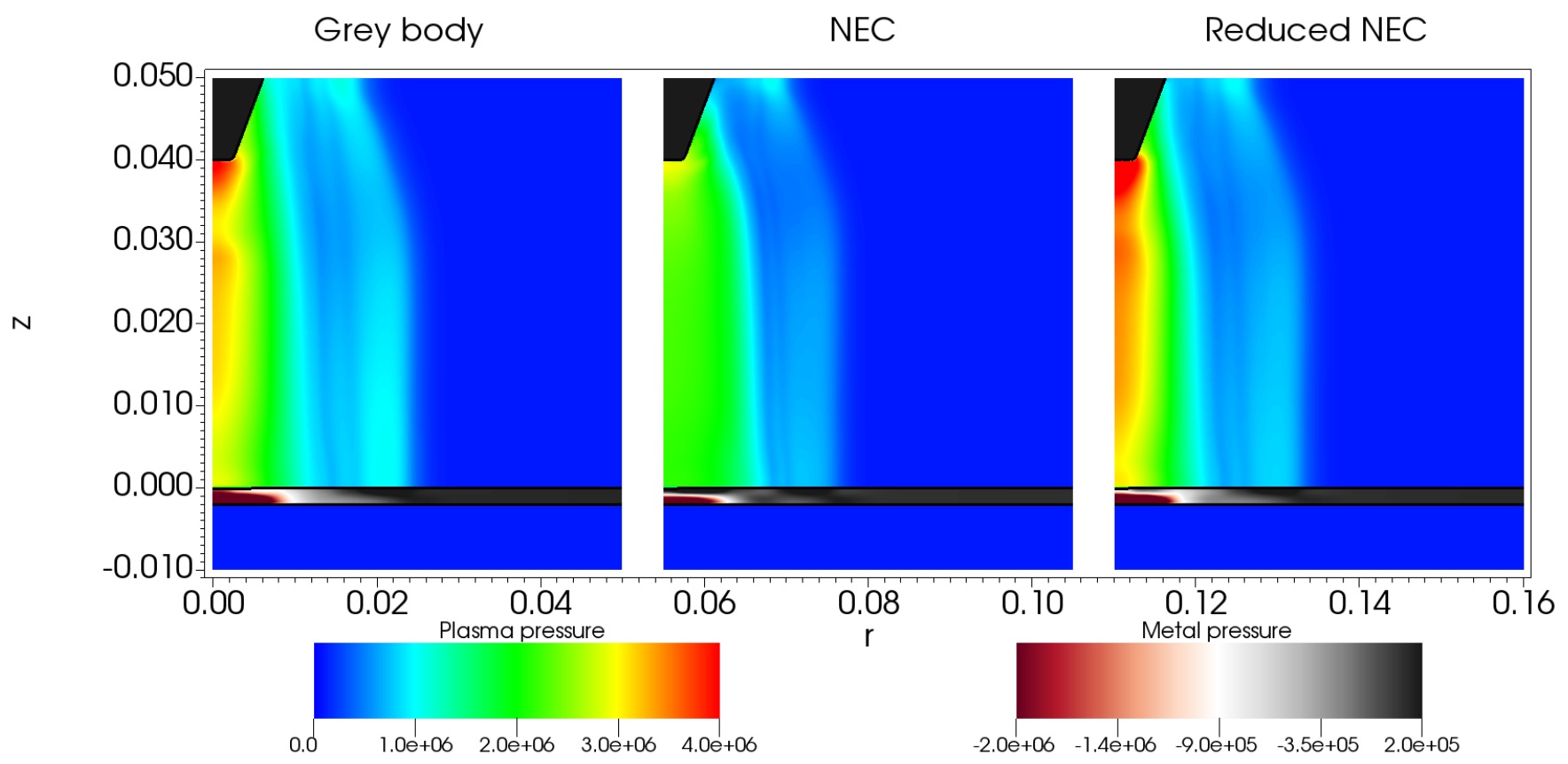

(a) Pressure

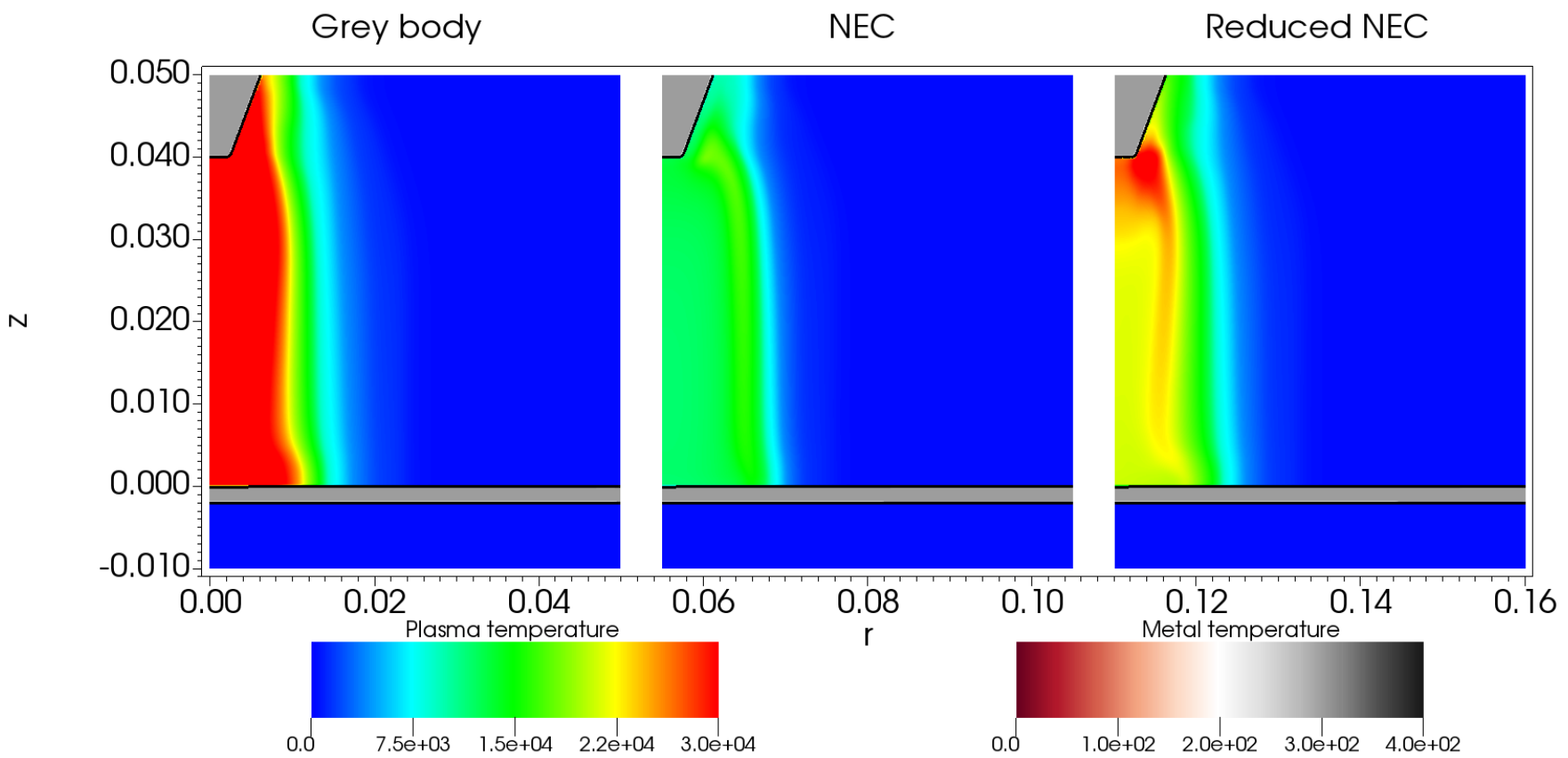

(b) Temperature

FIG. 4: Pressure and temperature profiles of the arc attachment to aluminium after $14 \mu \mathrm{s}$ of arc evolution showing how these vary betweeen the radiative models. The grey body model generates the highest temperature and pressure within the arc but the reduced NEC model produces slightly higher pressures in the aluminium plate which could lead to greater deformation.

It would also be of significant interest to apply this approach to attachment to composite materials, such that damage can be investigated. This requires additional development; composite materials are typically anisotropic, and would require a full three-dimensional model to accomplish this, complete with a suitable equation of state for the material, such as that

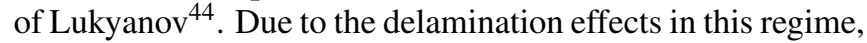
this would also require a damage model to simulate this behaviour.

In conclusion, the implementation of this equation of state- 


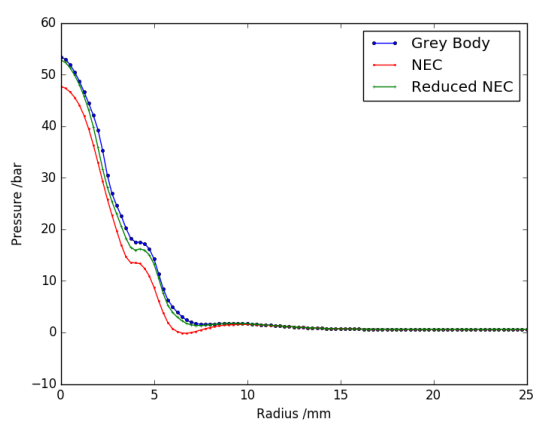

(a) $2 \mu \mathrm{s}$

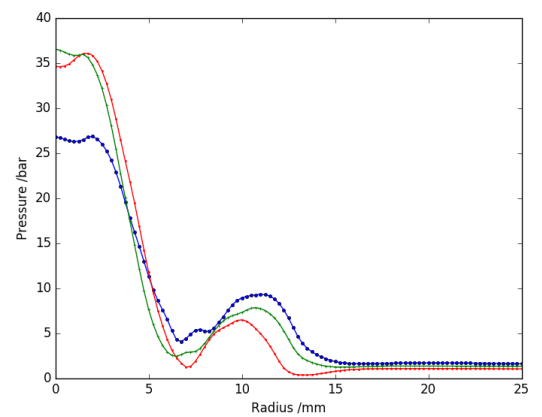

(c) $6 \mu \mathrm{s}$

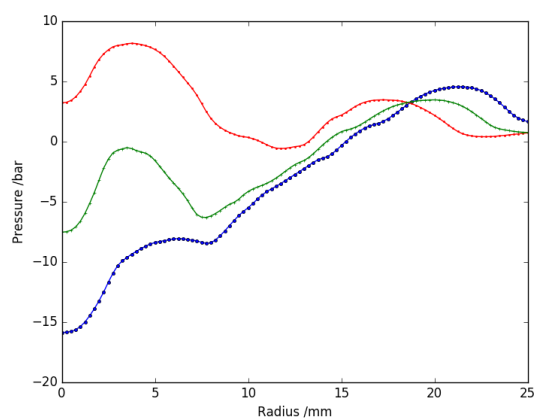

(e) $14 \mu \mathrm{s}$

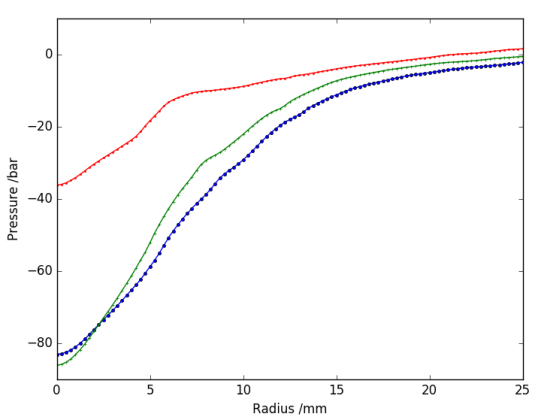

(g) $26 \mu \mathrm{s}$

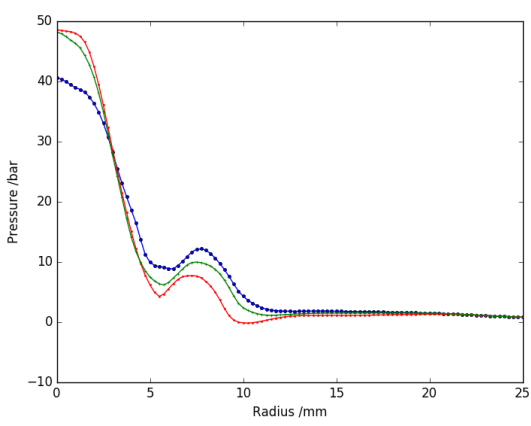

(b) $4 \mu \mathrm{s}$

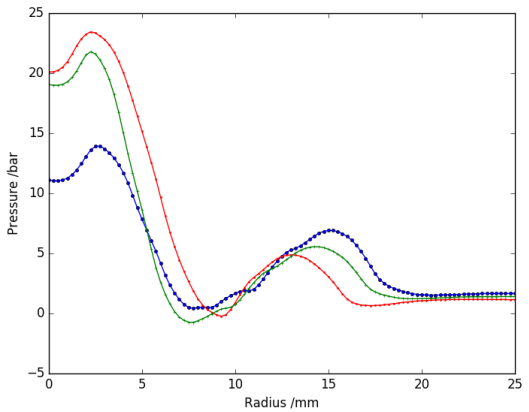

(d) $9 \mu \mathrm{s}$

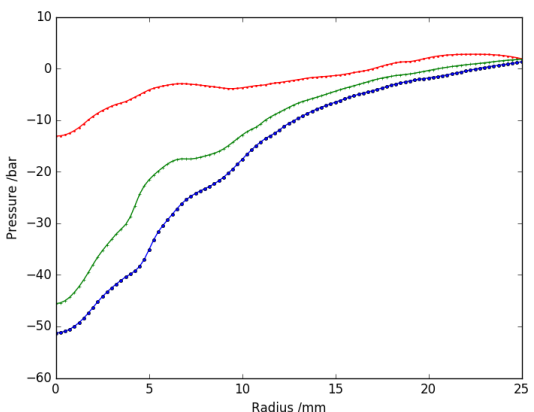

(f) $20 \mu \mathrm{s}$

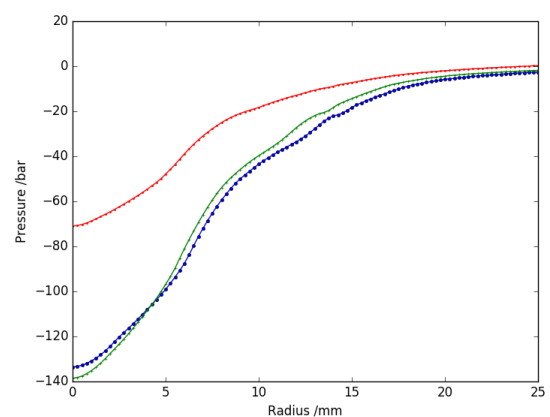

(h) $36 \mu \mathrm{s}$

FIG. 5: Pressure evolution at the surface of the aluminium plate for three radiation models for the attached arc. All three show similar behaviour; an initial rise in pressure, followed by a drop as the substrate is under tension. However, this behaviour occurs at different rates, with the grey body approach leading to the most rapid evolution of the pressure profile, and the NEC showing a much slower evolution. 
based radiative approach offers a reliable tool to support the design and validation of aeronautical materials under lightning strike conditions.

\section{ACKNOWLEDGMENTS}

M. Apsley acknowledges support by the UK Engineering and Physical Sciences Research Council (EPSRC) EP/L015552/1 for the Centre for Doctoral Training (CDT) in Computational Methods for Materials Science. The authors would also like to thank Micah Goldade of Boeing Research and Technology (BR\&T) for technical input throughout the work, and Carmen Guerra-Garcia (BR\&T and currently at the Massachusetts Institute of Technology) for suggestions on how best to model behaviours of a plasma.

\section{Appendix A: Absorption Coefficient}

An absorption coefficient has been developed for the 19species EoS used by this paper, taking into consideration electron transitions within species of up to one degree of ionisiation, based on the approach of Chauveau et al. 7 . The absorption coefficient, $\kappa$, is composed of contributions from freefree, bound-free, and bound-bound transitions 5 ,

$$
\kappa=\kappa^{\mathrm{bb}}+\kappa^{\mathrm{bf}}+\kappa^{\mathrm{ff}} .
$$

The bound-bound contributions produce spectral lines when electrons move between bound states in an atom or molecule, where the atomic lines depend on wavelength, $\lambda$, and temperature, $T$, accroding to ${ }^{1}$

$$
\begin{aligned}
\kappa^{b b}=\frac{\lambda^{5}\left(e^{h c / \lambda k_{B} T}-1\right)}{2 h c^{2}} \sum_{\text {line }} \frac{h c}{4 \pi}\left(\frac{g_{u} A_{u l}}{\lambda_{\text {line }}}\right) \\
\times \frac{N_{0}\left(T, N_{e}\right)}{Q(T)} e^{-E_{u} / k_{B} T} \phi\left(\lambda-\lambda_{\text {line }}, T, N_{e}\right)
\end{aligned}
$$

where $e$ is the charge on an electron, $h$ Planck's constant, $c$ the speed of light in a vacuum, $k_{B}$ the Boltzmann constant, $g_{u}$ ane $E_{u}$ the degeneracy and energy of the upper energy level, $A_{u l}$ the Einstein absorption coefficient for the transition, $N_{0}$ and $N_{E}$ the number densities of the radiating species and the electrons respectively and $\phi\left(\lambda-\lambda_{\text {line }}, T, N_{e}\right)$, is the spectral line shape. A spectral line always has a natural line width because there is a quantum-related uncertainty in the energy of the states involved, though for the scales considered in this work, the natural line width of a plasma, around $\sim 10^{-4} \mathrm{~nm}$, can be neglected. For an air plasma, the Stark effect due to collisions between species is the dominant line-broadening effect. Doppler shifts due to thermal motions of the atoms can also cause line broadening in the shape of a Gaussian distribution of half width at half maximum (HWHM) of $\sim 0.01 \mathrm{~nm}$ at temperatures of $40000 \mathrm{~K}$. This is approximately 20 times smaller than the HWHM of the Stark contribution, thus Doppler effects can be neglected. Also negligible are resonance and Van der Waals contributions which have HWHM proportional to the density of neutral perturbers which decreases sharply with temperature 45 .

The line shape of the Stark effect for a line at wavelength $\lambda_{\text {line }}$ and HWHM of $\gamma$ is approximated by a Lorentzian profile,

$$
\phi\left(\lambda-\lambda_{\text {line }}, T, N_{e}\right)=\frac{1}{\pi} \frac{\gamma}{\gamma^{2}+\left(\lambda-\lambda_{\text {line }}\right)^{2}} .
$$

In this work, four species were considered; $\mathrm{O}, \mathrm{N}, \mathrm{O}^{+}$, and $\mathrm{N}^{+}$. The data for $\mathrm{O}^{+}$and $\mathrm{N}^{+}$was taken from appendix $\mathrm{B}$ of Martins $\frac{1}{1}$, the line widths for $\mathrm{N}$ and $\mathrm{O}$ are tabulated in the work of Konjecvić and co-authors $4 \frac{48}{46}$, and the rest of the required data was taken from the NIST database 49 . The Stark line width was assumed to be proportional to number density of electrons and inversely proportional to the square root of temperature.

Bound-bound contributions for diatomic molecules take the form 50

$$
\kappa_{\mathrm{mol}}^{\mathrm{bb}}=N_{i} L \sum_{j} S_{i j} \phi_{i j}
$$

where $N_{i} L$ is the number density of energy level $i$ for each molecule. The line strength $S_{i j}$ of the transition from $i$ to $j$ is 51

$$
\begin{aligned}
S_{i j} & =\frac{A_{i j}}{8 \pi c v_{i j}^{2}} g^{\prime} \frac{e^{-c_{2} E^{\prime \prime} / T}\left(1-e^{-c_{2} v_{i j}}\right)}{Q(T)} \\
& =S_{i j}\left(T_{\text {ref }}\right) \frac{Q\left(T_{\text {ref }}\right)}{Q(T)} \frac{\exp \left(-c_{2} E^{\prime \prime} / T\right)}{\exp \left(-c_{2} E^{\prime \prime} / T_{\text {ref }}\right)} \\
& \times \frac{\left(1-\exp \left(-c_{2} v_{i j} / T\right)\right)}{\left(1-\exp \left(-c_{2} v_{i j} / T_{\text {ref }}\right)\right)}
\end{aligned}
$$

where $c_{2}=h c / k_{B}, Q(T)$ is the partition function, $E^{\prime \prime}$ is the energy of the lower level, $v_{i j}$ is the frequency of the transition and $A_{i j}$ is the Einstein emission coefficient. This is only strictly valid for temperatures near $T_{\text {ref }}=296 \mathrm{~K}$ however results obtained for temperatures up to $15000 \mathrm{~K}$ are sufficiently accurate for the purposes of this study and molecular boundbound contributions are only significant for $T<10000 \mathrm{~K}$. Chauveau et al ${ }^{7}$ show that for the NEC only $\mathrm{N}_{2}$, NO and $\mathrm{O}_{2}$ contribute. The data for $\mathrm{N}_{2}$ and $\mathrm{O}_{2}$ were obtained from the HITRAN molecular spectral database 51 . No data for NO could be found.

Next the free-free contributions were considered. For $\mathrm{N}^{+}$ and $\mathrm{O}^{+}$the emission coefficient for a plasma with ion density $N_{i}$ and electron density $N_{e}$ is

$$
\varepsilon_{v}^{\mathrm{ff}}=g(\tilde{v}, T) \frac{8}{3}\left(\frac{2 \pi}{3 k_{B} T m_{e}}\right)^{1 / 2} \frac{\alpha^{2} e^{6}}{m_{e} c^{3}} \exp \left(-\frac{h v}{k_{B} T}\right) N_{e} N_{i}
$$

where $\alpha$ is the fine structure constant and $m_{e}$ is the electron mass. For $T>11604 \mathrm{~K}$ the expression for the Gaunt factor, $g(\tilde{v}, T)^{52}$

$$
\begin{aligned}
g(\lambda, T) & =g_{c}(\lambda, T)\left[1+\varepsilon_{c}(\lambda, T)\right] \\
g_{c}(\lambda, T) & =1.270 \ln \left(0.2066 \lambda T^{3 / 2}\right) \\
\varepsilon_{c} & =-\left(7.4-\frac{45}{T}\right) \frac{1}{\lambda}+\left(4.9-\frac{18}{T}\right) \frac{100}{\lambda^{2}}
\end{aligned}
$$


was assumed. For $T<11604 \mathrm{~K}$ the Gaunt factor of Menzel and Pekeris $\sqrt{53}$ was used:

$$
g_{v}(v, T)=1+0.1728\left(\frac{v}{R_{H} Z^{2}}\right)^{1 / 3}\left[\frac{2}{\kappa^{2}}\left(\frac{R_{H} Z^{2}}{v}\right)-1\right],
$$

where $Z$ is the atomic number and $R_{H}$ is the Rydberg constant.

For $\mathrm{N}$ and $\mathrm{O}$, absorption occurs through inverse bremsstrahlung whereby free electrons absorb radiation. The absorption coefficient is then given by

$$
\begin{aligned}
& \kappa^{\mathrm{bb}}=8 \pi^{2}(2 \pi)^{1 / 2}\left(\frac{\hbar c}{e^{2}}\right)^{2} \frac{\left(e^{2} / a_{0}\right)^{3 / 2}\left(k_{B} T\right)^{3 / 2}}{(h v)^{3}} \\
& \times\left[1+\frac{h v}{2 k_{B} T}\right] a_{0}^{5} N N_{e}
\end{aligned}
$$

where $a_{0}$ is Bohr's constant and $\hbar=\frac{h}{2 \pi}$. This is valid for wavenumbers in the range 4800 to $24000 \mathrm{~cm}^{-1}$ and temperatures of 4000 to $12000 \mathrm{~K}$.

The molecular contributions to the free-free component also arise from inverse bremsstrahlung and depend on the cross section $\Sigma_{a}(\tilde{v}, T)$ according to

$$
\kappa^{\mathrm{ff}}=\Sigma_{a}(\tilde{v}, T) N_{m o l}\left[1-\exp \left(-\frac{h c}{\lambda k_{B} T}\right)\right]
$$

The contribution from molecular ions is negligible ${ }^{7}$ and so only $\mathrm{N}_{2}$ and $\mathrm{O}_{2}$ are taken into account. The data from Kive ${ }^{54}$, which is given for wavelengths of 0.3 to $4.8 \mu \mathrm{m}$ and temperatures of 3000 to $15000 \mathrm{~K}$, was interpolated within this range and extrapolated for $T<3000 \mathrm{~K}$ or wavenumbers, $\tilde{v}<$ $2083 \mathrm{~cm}^{-1}$.

The bound-free continuum absorption coefficient also has atomic and molecular contributions. For $\mathrm{N}, \mathrm{O}, \mathrm{N}^{+}$and $\mathrm{O}^{+}$it has the form

$$
\kappa^{\mathrm{bf}}=\left[\sum_{i} N_{i} \Sigma_{i}(\lambda)\right]\left[1-\exp \left(-\frac{h c \tilde{v}}{k_{B} T}\right)\right]
$$

where $g_{i}$ is the statistical weight of level $i$, and the population density of level $i$ depends on the total population density $N_{0}$ according to

$$
N_{i}=N_{0} \frac{g_{i}}{Q(T)} \exp \left(-\frac{\Delta E_{i}}{k_{B} T}\right)
$$

and the partition function is

$$
Q(T)=\sum_{i} g_{i} \exp \left(-\frac{\Delta E_{i}}{k_{B} T}\right) .
$$

The transition energies, $\Delta E$, and cross sections, $\Sigma_{i}$, are taken from the TOPBASE database 55 .

For $\mathrm{O}^{-}$and $\mathrm{N}^{-}$photoabsorption by the ${ }^{3} P$ and ${ }^{2} P$ levels respectively dominates with population densities also given by equation (A11). The energy levels for $\mathrm{N}^{-}$can be found in Cowan et al. ${ }^{56}$ and for $\mathrm{O}^{-}$they are given by Garrett and Jackson Jr. ${ }^{[57}$. The absorptivity is then

$$
\kappa_{\sigma}=N \Sigma(\lambda)\left[1-\exp \left(-\frac{h c}{\lambda k_{B} T}\right)\right]
$$

where for $\mathrm{N}^{-}$

$$
\Sigma= \begin{cases}2 \times 10^{-17} & \text { if } \tilde{v} \geq 806.55 \mathrm{~cm}^{-1} \\ 0 & \text { Otherwise }\end{cases}
$$

and for $\mathrm{O}^{-}$the values of $\Sigma$ come from ${ }^{7}$.

The molecular contributions, from $\mathrm{N}_{2}, \mathrm{O}_{2}$ and $\mathrm{NO}$, have the same form as for photoabsorption. In the case of $\mathrm{N}_{2}$ and $\mathrm{O}_{2}$ the cross sections are taken from Fennelly and Tor ${ }^{58}$. For NO, an analytical expression can be found in Romanov et al.59. The data determining the shape of the Schumann-Runge continuum, the longest absorption band in molecular oxygen, are taken from Churchill et al. ${ }^{60}$ for temperatures in the range 300 to $1000 \mathrm{~K}$ and wavenumbers of 57000 to $77000 \mathrm{~cm}^{-1}$. This is extrapolated linearly down to $\tilde{v}=48000 \mathrm{~cm}^{-1}$.

The atomic bound-bound contributions were validated against results from Martins ${ }^{1}$ in figure 6 Sample results are shown for $N_{e}=3 \times 10^{18} \mathrm{~cm}^{-3}$ at temperatures of $25 \mathrm{kK}$, $30 \mathrm{kK}$, and $35 \mathrm{kK}$. The results match well, except at $35 \mathrm{kK}$ where the peaks coincide but the troughs are deeper. This may be due to the difference in number density between the estimated value and the true value used by Martins.

To validate the continuum absorption coefficients, the bound-free and bound-bound contributions are plotted in figure 7 for temperatures of 2000, 8000 and $15000 \mathrm{~K}$ against the results obtained by Chauveau et al. ${ }^{7}$. Molar densities from the NASA CEA database 61 are used. They agree well and only diverge significantly at wavelengths where the contributions are no longer significant.

Figure 8 shows the total absorption coefficient. The results compare well to those of Chauveau et al 62 though there are some differences due to the fact that the HITRAN database does not include data for as many lines as are considered by Chauveau et al. and consequently some of the SchumannRunge bands are missing at $2000 \mathrm{~K}$.

${ }^{1}$ R. S. Martins, Étude expérimentale et théorique d'un arc de foudre et son interaction avec un matériau aéronautique, Ph.D. thesis, Université ParisSaclay (2016).

${ }^{2}$ L. Chemartin, P. Lalande, B. Peyrou, A. Chazottes, P. Elias, C. Delalondre, B. Cheron, and F. Lago, "Direct effects of lightning on aircraft structure: analysis of the thermal, electrical and mechanical constraints," AerospaceLab , p-1 (2012).

${ }^{3}$ J. Zhang, X. Zhang, X. Cheng, Y. Hei, L. Xing, and Z. Li, "Lightning strike damage on the composite laminates with carbon nanotube films: Protection effect and damage mechanism," Composites Part B: Engineering 168, 342 352 (2019).

${ }^{4}$ J. Lowke, "A relaxation method of calculating arc temperature profiles applied to discharges in sodium vapor," Journal of Quantitative Spectroscopy and Radiative Transfer 9, 839-854 (1969).

${ }^{5} \mathrm{~J}$. Lowke and E. Capriotti, "Calculation of temperature profiles of high pressure electric arcs using the diffusion approximation for radiation transfer," Journal of Quantitative Spectroscopy and Radiative Transfer 9, 207-236 (1969).

${ }^{6}$ W. Hermann and E. Schade, "Radiative energy balance in cylindrical nitrogen arcs," Journal of Quantitative Spectroscopy and Radiative Transfer 12, 1257-1282 (1972).

${ }^{7}$ S. Chauveau, C. Deron, M.-Y. Perrin, P. Riviere, and A. Soufiani, "Radiative transfer in LTE air plasmas for temperatures up to 15; $000 \mathrm{~K}$," Journal of Quantitative Spectroscopy \&Radiative Transfer 77, 113-130 (2003).

${ }^{8} \mathrm{Y}$. Cressault, "Basic knowledge on radiative and transport properties to begin in thermal plasmas modelling," AIP Advances 5, 057112 (2015). 

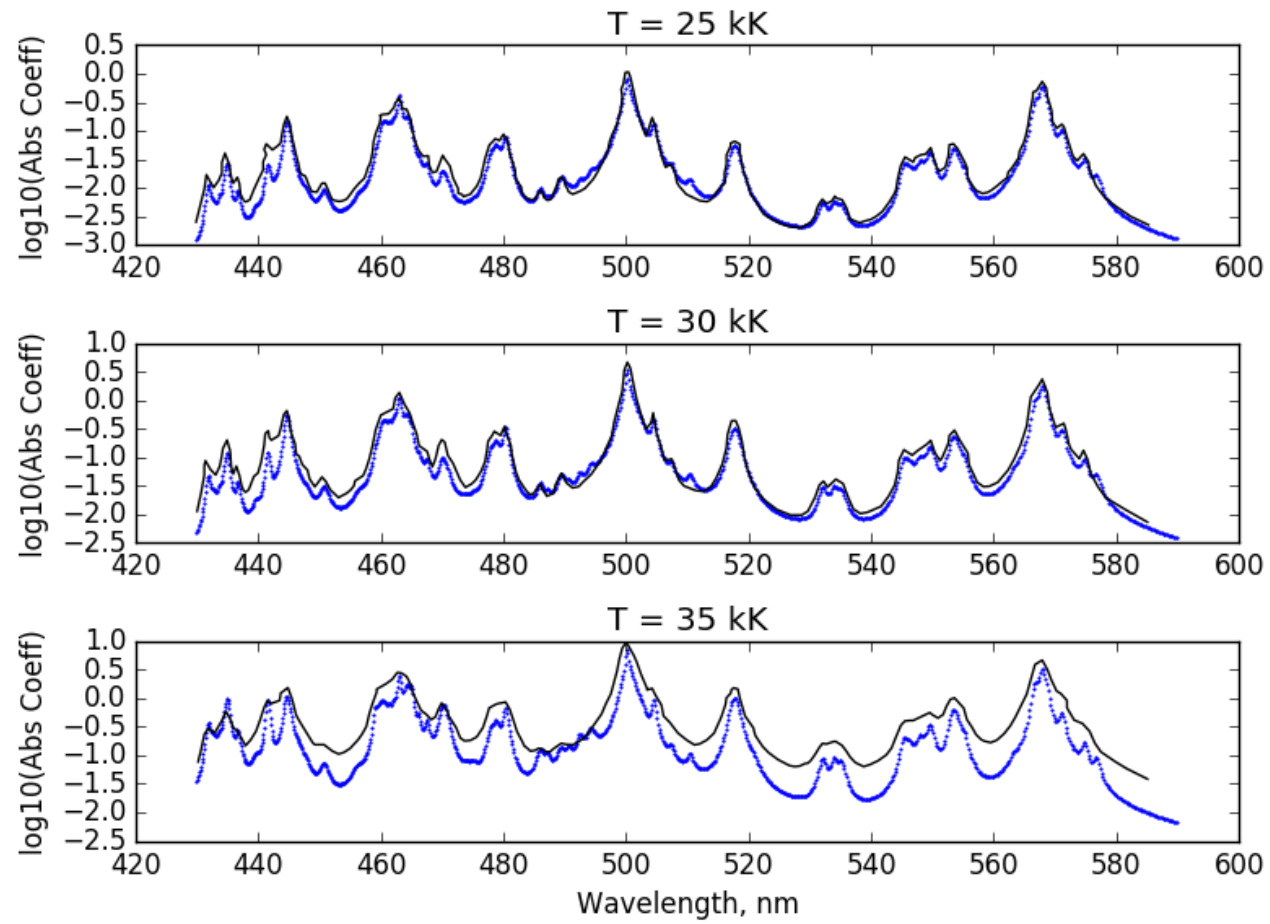

FIG. 6: Bound-bound absorption coefficient $\left(\mathrm{cm}^{-1}\right)$ for $\mathrm{N}^{+}$and $\mathrm{O}^{+}$for $N_{e}=3 \times 10^{18} \mathrm{~cm}^{-3}$ for temperatures of $25 \mathrm{kK}, 30 \mathrm{kK}$, and $35 \mathrm{kK}$; calculated results in blue, results from Martins ${ }^{1}$ in black. These show good agreement, especially for lower arc temepratures, though some differences in absorption are visible for higher temperatures.

${ }^{9}$ J. Lowke, "Predictions of arc temperature profiles using approximate emission coefficients for radiation losses," J. Qumt. Specrrosc. Rmfiat. Trans/er. 14, 111-122 (1974)

${ }^{10} \mathrm{G}$. Raynal and A. Gleizes, "Radiative transfer calculation in SF6 arc plasmas using partial characteristics," Plasma Sources Science and Technology 4, 152 (1995).

${ }^{11} \mathrm{~S}$. Eby, J. Trepanier, and X. Zhang, "Modelling radiative transfer in circuitbreaker arcs with the P-1 approximation," Journal of Physics D: Applied Physics 31, 1578 (1998).

${ }^{12}$ L. Chemartin, P. Lalande, E. Montreuil, C. Delalondre, B. Cheron, and F. Lago, "Three dimensional simulation of a DC free burning arc. Application to lightning physics," Atmospheric Research 91, 371-380 (2009).

${ }^{13}$ P. Teulet, T. Billoux, Y. Cressault, M. Masquère, A. Gleizes, I. Revel, B. Lepetit, and G. Peres, "Energy balance and assessment of the pressure build-up around a bolt fastener due to sparking during a lightning impact," The European Physical Journal Applied Physics 77, 20801 (2017).

${ }^{14}$ W. Fusheng, M. Xiangteng, C. Han, and Y. Zhang, "Evolution simulation of lightning discharge based on a magnetohydrodynamics method," Plasma Science and Technology 20, 075301 (2018).

${ }^{15}$ C. Da Silva, R. Sonnenfeld, H. Edens, P. Krehbiel, M. Quick, and W. Koshak, "The plasma nature of lightning channels and the resulting nonlinear resistance," Journal of Geophysical Research: Atmospheres 124, 9442-9463 (2019).

${ }^{16}$ J. Lacombe, Y. Delannoy, and C. Trassy, "The role of radiation in modelling of argon inductively coupled plasmas at atmospheric pressure," Journal of Physics D: Applied Physics 41, 165204 (2008).

${ }^{17}$ N. Aleksandrov, E. Bazelyan, and M. Shneider, "Effect of continuous current during pauses between successive strokes on the decay of the lightning channel," Plasma Physics Reports 26, 893-901 (2000).

${ }^{18} \mathrm{~F}$. Tholin, L. Chemartin, and P. Lalande, "Numerical investigation of the interaction of a lightning and an aeronautic skin during the pulsed arc phase," IET Conference Proceedings , 39 (6 .)-39 (6 .)(1) (2015)

${ }^{19}$ B. Peyrou, L. Chemartin, P. Lalande, B. Chéron, P. Riviere, M. Perrin, and A. Soufiani, "Radiative properties and radiative transfer in high pressure thermal air plasmas," Journal of Physics D: Applied Physics 45, 455203 (2012).

${ }^{20}$ M. Bartlova, P. Kloc, N. Bogatyreva, V. Aubrecht, and J. Pokorny, "Modelling of radiative transfer in air arc plasma," Plasma Physics and Technology 4, 261-264 (2017).

${ }^{21}$ S. Eby, J. Trepanier, and X. Zhang, "Modelling radiative transfer in circuitbreaker arcs with the p-1 approximation," Journal of Physics D: Applied Physics 31, 1578 (1998).

${ }^{22} \mathrm{H}$. Nordborg and A. Iordanidis, "Self-consistent radiation based modelling of electric arcs: I. efficient radiation approximations," Journal of Physics D: Applied Physics 41, 135205 (2008).

${ }^{23}$ V. Sevast'yanenko, "Radiation transfer in a real spectrum. Integration over frequency," Journal of Engineering Physics and Thermophysics 36, 138148 (1979).

${ }^{24} \mathrm{~V}$. Sevast'yanenko, "Radiation transfer in a real spectrum. Integration with respect to the frequency and angles," Journal of engineering physics 38, 173-179 (1980).

${ }^{25}$ T. Gogel, A. Sedghinasab, and D. Keefer, "Radiation transfer computation in cylindrical arc columns using a monte carlo method," Journal of Quantitative Spectroscopy and Radiative Transfer 52, 179-194 (1994).

${ }^{26}$ A. Villa, R. Malgesini, and L. Barbieri, "A multiscale technique for the validation of a numerical code for predicting the pressure field induced by a high-power spark," Journal of Physics D: Applied Physics 44, 165201 (2011).

${ }^{27}$ S. Millmore and N. Nikiforakis, "Multi-physics simulations of lightning strike on elastoplastic substrates," Journal of Computational Physics 405, $109142(2020)$

${ }^{28}$ F. Träuble, S. Millmore, and N. Nikiforakis, "An improved equation of state for air plasma simulations," Physics of Fluids 33, 036112 (2021).

${ }^{29}$ Michael Louisa, Millmore Stephen T., and Nikiforakis Nikolaos, "A Multi-physics Methodology for Four States of Matter," Communications on Applied Mathematics and Computation (2019), https://doi.org/10.1007/s42967-019-00047-4

${ }^{30}$ Y. Tanaka, T. Michishita, and Y. Uesugi, "Hydrodynamic chemical non- 


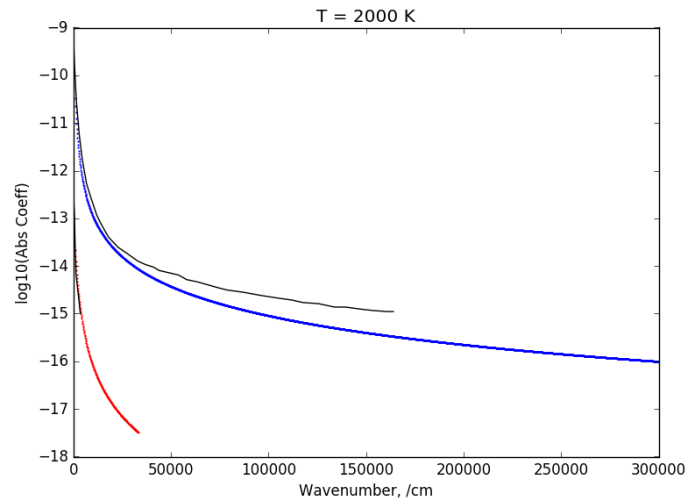

(a)

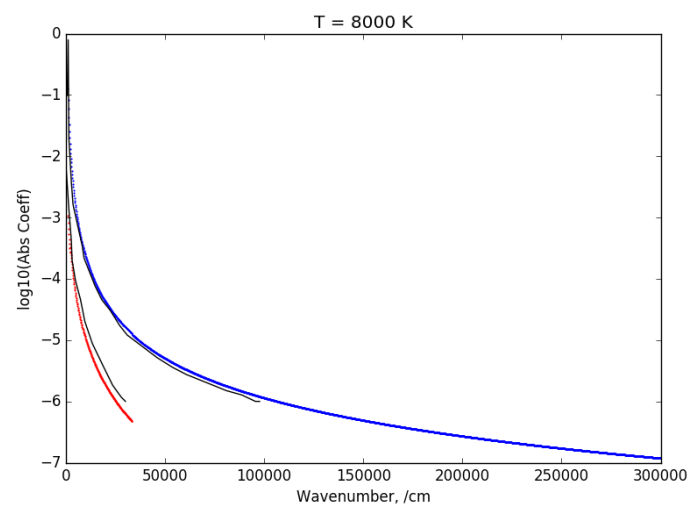

(c)

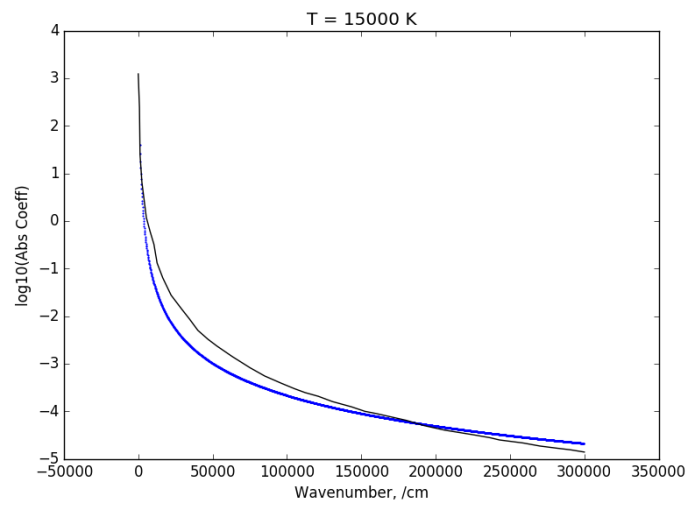

(e)

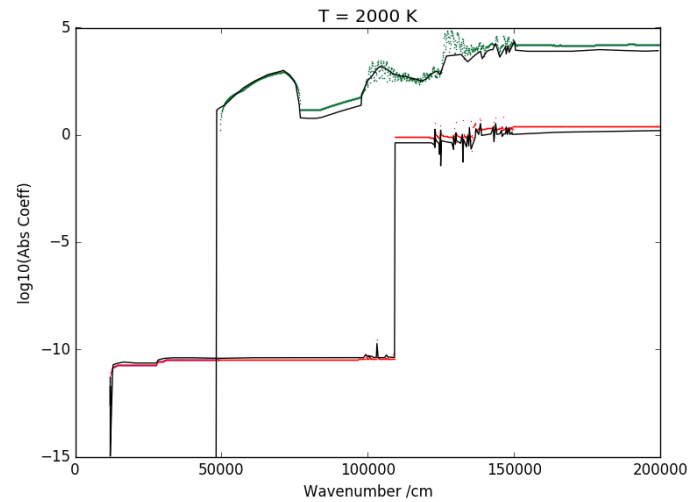

(b)

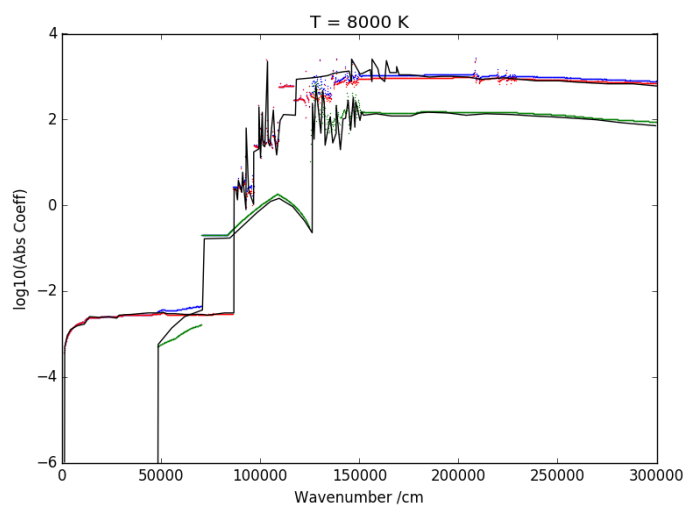

(d)

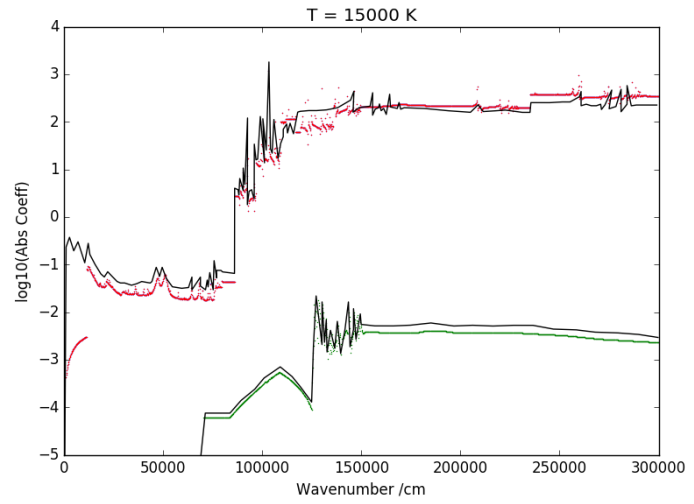

(f)

FIG. 7: Free-free (left) and bound-free (right) contributions (molecular in red, atomic in blue) compared to results of Chauveau et al.$^{7}$ at (top) $2000 \mathrm{~K}$, (middle) $8000 \mathrm{~K}$, (bottom) $15000 \mathrm{~K}$. Comparable values for the absorption coefficinet are visible, though at higher temperatures, some peaks are missing.

equilibrium model of a pulsed arc discharge in dry air at atmospheric pressure," Plasma Sources Science and Technology 14, 134 (2005).

${ }^{31}$ A. Gleizes, J.-J. Gonzalez, and P. Freton, "Thermal plasma modelling," Journal of Physics D: Applied Physics 38, R153 (2005).

${ }^{32}$ J. Haidar, "Non-equilibrium modelling of transferred arcs," Journal of Physics D: Applied Physics 32, 263 (1999).

${ }^{33}$ S. ARP5412A, "Aircraft lightning environment and related test waveforms," Society of Automotive Engineers, Aerospace Recommended Practice, PA, Revised 2 (2005).
${ }^{34}$ J. T. Tables, "Parts I and II," J. Phys. Chem. Ref. Data 14 (1985), https : //janaf.nist.gov

${ }^{35}$ B. Ruscic, "Active Thermochemical Tables (ATcT) values based on ver. 1.122 of the Thermochemical Network, 2015," available at ATcT. anl. gov (2017), https://atct.anl.gov/Thermochemical\%20Data/ version\%201.122/index.php

${ }^{36}$ M. Winter, "WebElements," (2018), https ://www . webelements . com/ nitrogen/atoms.html

${ }^{3 /} \mathrm{G}$. Raynal and A. Gleizes, "Radiative transfer calculation in SF 6 arc plas- 


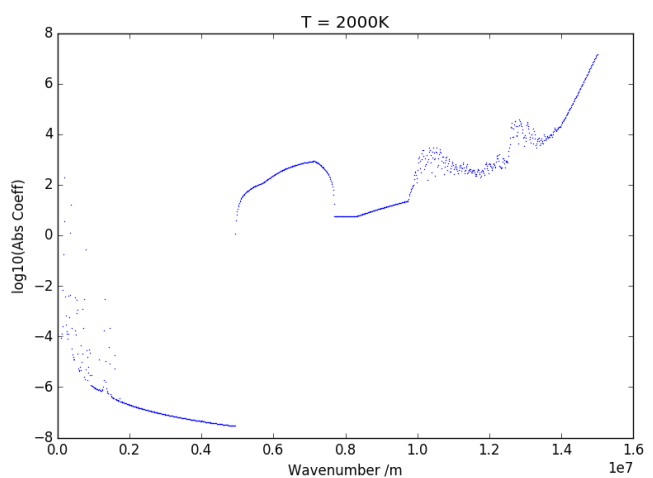

(a)



(b)

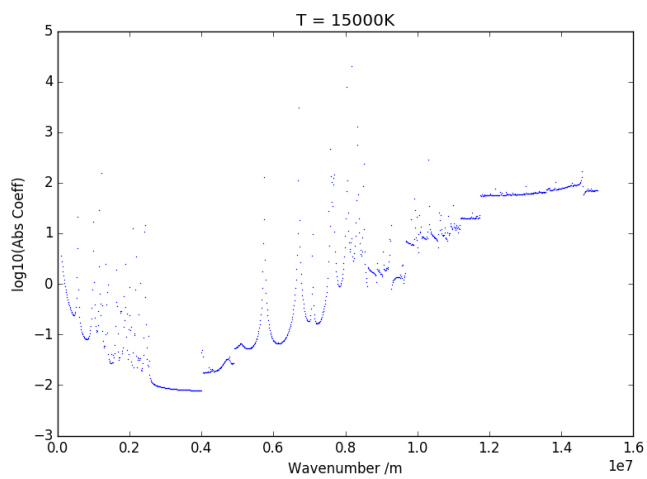

(c)

FIG. 8: Total absorption coefficient (left) at 1 atm (top) $2000 \mathrm{~K}$, (middle) $8000 \mathrm{~K}$, (bottom) $15000 \mathrm{~K}$ in all three cases, these results compare well with those of Chauveau et al ${ }^{7}$. Although some structures, including the

Schumann-Runge bands, are not present in these results, the comparable shape and peak values suggest that this is a reasonable model for absorption coefficient.

mas using partial characteristics," Plasma Sources Sci. Technol. 4, 152-160 (1995).

${ }^{38}$ E. L. Wachspress, "Trail to a lyapunov equation solver," Computers \& Mathematics with Applications 55, 1653-1659 (2008).

${ }^{39}$ E. W. Larsen, G. Thömmes, A. Klar, M. Seaïd, and T. Götz, "Simplified P $\mathrm{N}$ Approximations to the Equations of Radiative Heat Transfer and Applications," Journal of Computational Physics 183, 652-675 (2002).
${ }^{40}$ Y. Naghizadeh-Kashani, Y. Cressault, and A. Gleizes, "Net emission coefficient of air thermal plasmas," Journal of Physics D: Applied Physics 35, 2925 (2002).

${ }^{41}$ K. Hsu, K. Etemadi, and E. Pfender, "Study of the free-burning highintensity argon arc," Journal of applied physics 54, 1293-1301 (1983).

${ }^{42}$ L. Chemartin, P. Lalande, C. Delalondre, B. Cheron, and F. Lago, "Modelling and simulation of unsteady dc electric arcs and their interactions with electrodes," Journal of Physics D: Applied Physics 44, 194003 (2011).

${ }^{43}$ S. Kamiyama, Y. Hirano, and T. Ogasawara, "Delamination analysis of cfrp laminates exposed to lightning strike considering cooling process," Composite Structures 196, 55-62 (2018)

${ }^{44}$ A. A. Lukyanov, "An equation of state of a carbon-fibre epoxy composite under shock loading," The European Physical Journal B-Condensed Matter and Complex Systems 74, 35-45 (2010).

${ }^{45} \mathrm{~V}$. Aubrecht and M. Bartlova, "Net emission coefficients of radiation in air and SF 6 thermal plasmas," Plasma Chemistry and Plasma Processing 29, 131-147 (2009).

${ }^{46}$ N. Konjević, A. Lesage, J. R. Fuhr, and W. L. Wiese, "Experimental Stark widths and shifts for spectral lines of neutral and ionized atoms (a critical review of selected data for the period 1989 through 2000)," Journal of Physical and Chemical Reference Data 31, 819-927 (2002).

${ }^{47} \mathrm{~N}$. Konjevic and J. Roberts, "A critical review of the Stark widths and shifts of spectral lines from non-hydrogenic atoms," Journal of Physical and Chemical Reference Data 5, 209-257 (1976).

${ }^{48}$ N. Konjević, M. Dimitrijević, and W. Wiese, "Experimental Stark widths and shifts for spectral lines of neutral atoms (a critical review of selected data for the period 1976 to 1982)," Journal of physical and chemical reference data 13, 619-647 (1984).

${ }^{49}$ A. Kramida, Yu. Ralchenko, J. Reader, and and NIST ASD Team, NIST Atomic Spectra Database (ver. 5.5.6), [Online]. Available: https: //physics.nist.gov/asd [2018, May 30]. National Institute of Standards and Technology, Gaithersburg, MD. (2018).

${ }^{50} \mathrm{~K}$. Owen, Measurements of vibrational relaxation and dissociation of oxygen with laser absorption spectroscopy with applications for energy transfer in nonequilibrium air, Ph.D. thesis, Stanford University (2014).

${ }^{51}$ I. E. Gordon, L. S. Rothman, C. Hill, R. V. Kochanov, Y. Tan, P. F. Bernath, M. Birk, V. Boudon, A. Campargue, K. Chance, et al., "The HITRAN2016 molecular spectroscopic database," Journal of Quantitative Spectroscopy and Radiative Transfer 203, 3-69 (2017).

${ }^{52} \mathrm{~J}$. Stallcop and K. Billman, "Analytical formulae for the inverse bremsstrahlung absorption coefficient," Plasma Physics 16, 1187 (1974).

${ }^{53}$ D. H. Menzel and C. L. Pekeris, "Absorption coefficients and hydrogen line intensities," Monthly Notices of the Royal Astronomical Society 96, 77 (1935)

${ }^{54}$ B. Kivel, "Bremsstrahlung in air," Journal of Quantitative Spectroscopy and Radiative Transfer 7, 51-60 (1967).

${ }^{55}$ T. O. P. Team, "The Opacity Project," (1995).

${ }^{56}$ R. Cowan, C. F. Fischer, J. E. Hansen, and V. Kempter, "Excited states of the negative nitrogen ion: energies and lifetimes," Journal of Physics B: Atomic, Molecular and Optical Physics 30, 1457 (1997).

${ }^{57} \mathrm{~W}$. Garrett and H. Jackson Jr, "Electron photodetachment from O- and elastic scattering from atomic oxygen," Physical Review 153, 28 (1967).

${ }^{58} \mathrm{~J}$. Fennelly and D. Torr, "Photoionization and photoabsorption cross sections of $\mathrm{O}, \mathrm{N} 2, \mathrm{O} 2$, and $\mathrm{N}$ for aeronomic calculations," Atomic Data and Nuclear data tables 51, 321-363 (1992).

${ }^{59}$ G. Romanov, Y. A. Stankevich, L. Stanchits, and K. Stepanov, "Thermodynamic and optical properties of gases in a wide range of parameters," International journal of heat and mass transfer 38, 545-556 (1995).

${ }^{60}$ D. Churchill, B. Armstrong, R. Johnston, and K. Müller, "Absorption coefficients of heated air: a tabulation to $24000 \mathrm{~K}$," Journal of Quantitative Spectroscopy and Radiative Transfer 6, 371-442 (1966).

${ }^{61}$ B. J. McBride and S. Gordon, "Nasa reference publication 1311: computer program for calculation of complex chemical equilibrium compositions and applications, ii. user manual and program description," (1996).

${ }^{62}$ S. Chauveau, M.-Y. Perrin, P. Riviere, and A. Soufiani, "Contributions of diatomic molecular electronic systems to heated air radiation," Journal of Quantitative Spectroscopy and Radiative Transfer 72, 503-530 (2002). 